\title{
Adaptive and Robust Aeroelastic Control of Nonlinear Lifting Surfaces with Single/Multiple Control Surfaces: A Review
}

\author{
Z. Wang* and A. Behal** \\ Department of Electrical Engineering and Computer Science, University of Central Florida, NanoScience Technology Center, \\ University of Central Florida, Orlando, FL, 32816
}

\author{
P. Marzocca*** \\ Mechanical and Aeronautical Engineering, Clarkson University, Potsdam, NY, 1369
}

\begin{abstract}
Active aeroelastic control is an emerging technology aimed at providing solutions to structural systems that under the action of aerodynamic loads are prone to instability and catastrophic failures, and to oscillations that can yield structural failure by fatigue. The purpose of the aeroelastic control among others is to alleviate and even suppress the vibrations appearing in the flight vehicle subcritical flight regimes, to expand its flight envelope by increasing the flutter speed, and to enhance the postflutter behavior usually characterized by the presence of limit cycle oscillations. Recently adaptive and robust control strategies have demonstrated their superiority to classical feedback strategies. This review paper discusses the latest development on the topic by the authors. First, the available control techniques with focus on adaptive control schemes are reviewed, then the attention is focused on the advanced single-input and multi-input multi-output adaptive feedback control strategies developed for lifting surfaces operating at subsonic and supersonic flight speeds. A number of concepts involving various adaptive control methodologies, as well as results obtained with such controls are presented. Emphasis is placed on theoretical and numerical results obtained with the various control strategies.
\end{abstract}

Key words: Nonlinear active aeroelastic control, MIMO adaptive, robust and neural control, feedback and feedforward control design, aeroelasticity

\section{Nomenclature}

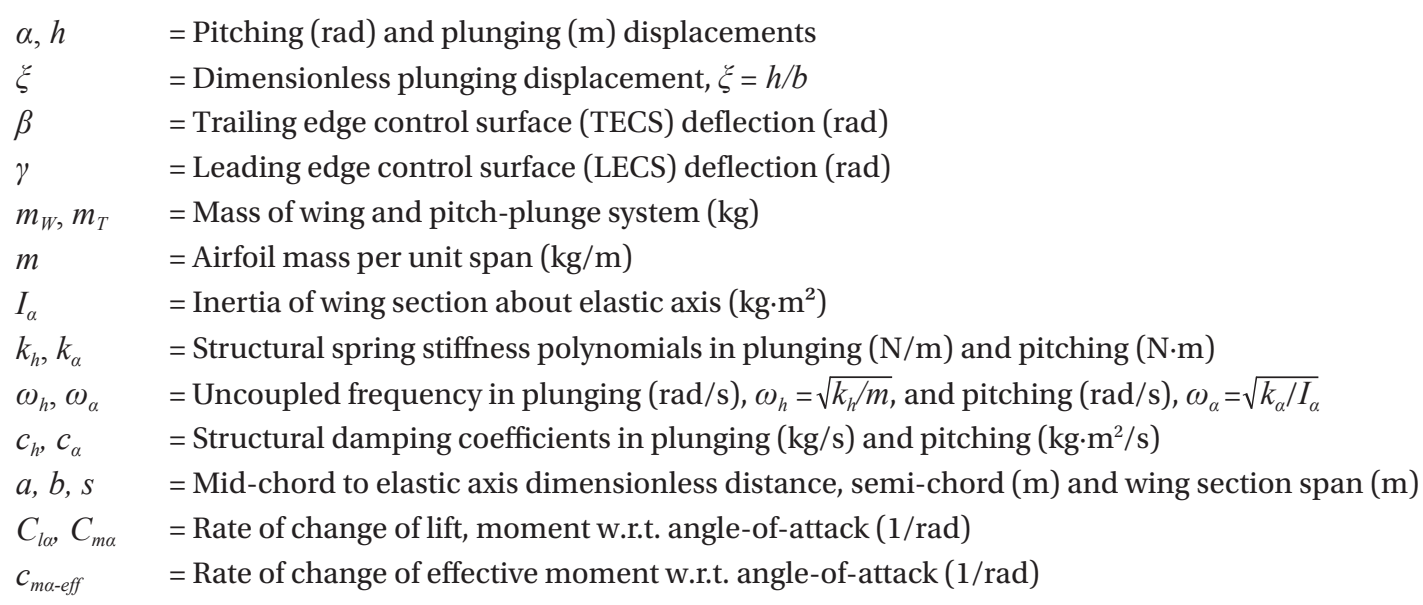




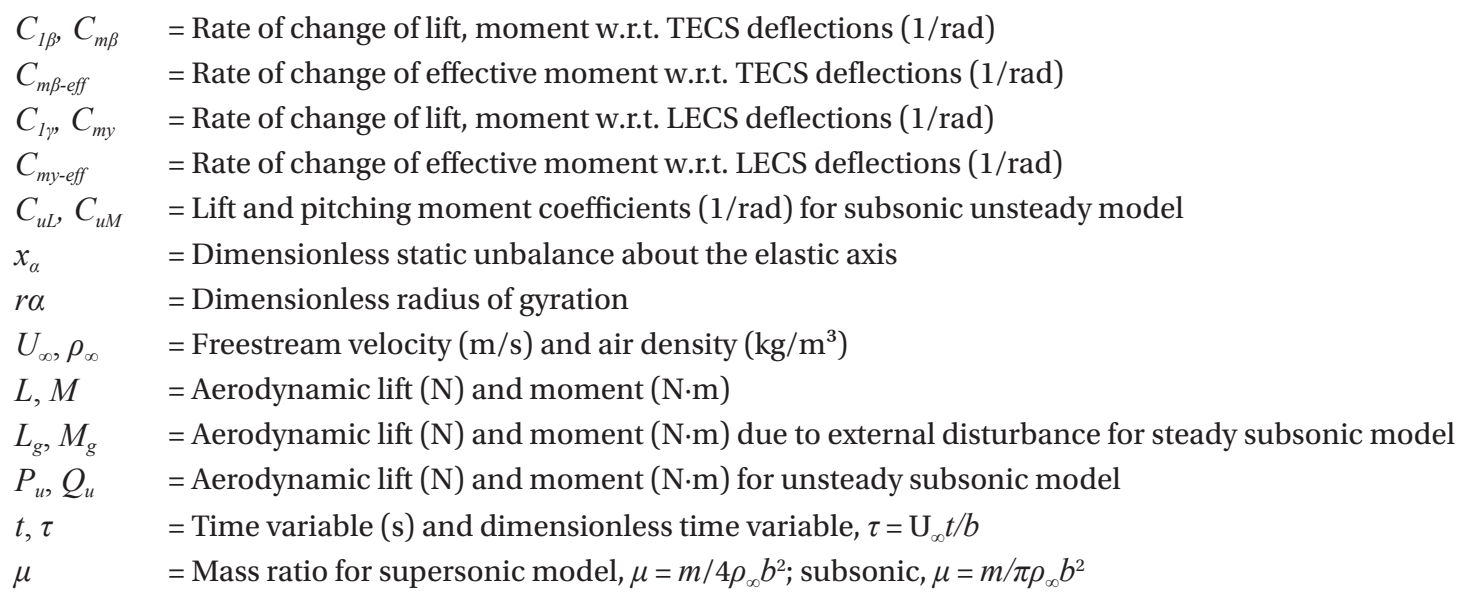

\section{Introduction}

The development and application of passive and active feedback control are current focus of research for aerospace, civil, and mechanical structural systems. Aircraft flight vehicles, long span bridges, wind turbines, skyscrapers, and Micro-Electro-Mechanical Systems (MEMS) are just a few examples of systems where passive and active feedback control is and will play a critical role in these technology development. Particularly, it's well known that the implementation of active feedback control design aimed to solve the aeroelastic instabilities problem, which can yield instantaneous catastrophic failure, e.g., flutter, or structural fatigue failure, due to persistent oscillations, e.g., limit cycle oscillations (LCOs) (Librescu and Marzocca, 2005), are emerging aeronautical and aerospace fight vehicles technologies. Since above dynamic aeroelastic effects are very likely to impose critical constraints on the flight vehicle performance, passive or active feedback control strategies need to be applied in order to safely perform maneuvers in the proximity of the flight envelope boundary. Although one can postpone the aeroelastic instabilities to provide an expansion of the flight envelope via increasing the stiffness of the wing section, the corresponding by-product of adding weight on the wing indeed decreased the overall performance of the flight vehicle over the entire flight envelope. Instead of simply increasing the stiffness of the wing section, active suppression mechanisms could yield improved performance with many successful implementation examples on suppressing flutter, divergence, control reversal, and detrimental wing oscillations, through feedback to control surfaces. An early comprehensive review of active control algorithms, windtunnel experiments, and flight experiences associated with feedback control and aeroelasticity was presented in Noor and Venneri (1992). Lyons et al. (1973) presented a theoretical study of flutter suppression under full-state feedback by utilizing Kalman estimator. Later, more complex higherorder aeroelastic models were subsequently developed. A methodology for designing optimal feedback control laws has been developed and implemented to active aeroelastic vibration suppression problems by Mukhopadhyay et al. (1980). Based on Linear Quadratic Gaussian (LQG) methodology, Gangsaas et al. (1981) proposed a gust load alleviation method and flutter suppression control laws. In both control strategies, output feedback control was used while the unmeasurable states were described by estimators. Karpel (1982) developed a partial-state feedback control law with pole placement techniques while Horikawa and Dowell (1979) adopted proportional gain feedback methods to achieve flutter suppression and load alleviation by directly feeding one of four variables back to the control surface. For the experimental results on the flutter suppression, by utilizing a simple but efficient proportional gain feedback control laws derived from root locus plots, Heeg (1993) has demonstrated that the flutter velocity of a bending-torsion spring mounted small wing model can be increased by up to 20\%. Results in Lin (1993) and Lazarus (1992) demonstrated that the proposed full-state feedback active control mechanism with an estimator was efficient by using a typical section with leading- and trailing-edge flaps. The disturbance rejection, gust alleviation, and flutter suppression were also demonstrated in the experimental investigations.

Although aforementioned results have shown the successful applications of the linear control theory in the aeroelastic instability problem, the need for more sophisticated aeroservoelastic models and control strategies stem from the fact that aircraft shows complex dynamical behaviors due to the presence of system nonlinearities under 
complex flight conditions. Recently, a significant amount of research in advancing methods for nonlinear aeroelasticity have been carried out, especially to deal with the softening or hardening structural stiffness effects and aerodynamic nonlinearities in the transonic or stall region. Dowell and Clark's book (2004) shed some lights on the importance of considering nonlinearities in the aeroelastic analysis. Specifically, hardening structural nonlinearities due to the presence of freeplay gave rise to nonlinear stiffness, especially in torsion. Usually this type of nonlinearity could yield a pronounced effect and lead to a limited amplitude aeroelastic behavior, which was not catastrophic but may finally lead to fatigue problems on the wing section (Breitbach, 1978; Eastep and Olsen, 1980; Edwards et al., 1983; Strganac and Mook, 1990; Yang and Zhao, 1988).

A review of recent work on the active control of aircraft wing with nonlinearity can be found in (Mukhopadhyay, 2000a, b, 2001). Several control algorithms have been tested on a typical wing section model using single control surface and examined experimentally (Behal et al., 2006a; Ko et al., 1997, 1998, 1999; Lee and Singh, 2007, 2009; Mukhopadhyay, 1992; Singh and Wang, 2002; Strganac et al., 2000; Vipperman et al., 1998; Wazak and Srinathkumar, 1992; Xing and Singh, 2000; Zhang and Soffker, 2009; Zhang and Singh, 2001), by using traditional root locus and Nyquist method, LQG method, nonlinear control theory as well as robust and adaptive control strategies. In general, the pitching angle $(\alpha)$ was considered as the primary output variable aimed to regulate to zero. Firstly, the nonlinearities introduced by the torsional stiffness were canceled out using adaptation, leading to a feedback linearized system. Secondly, full-state feedback control aimed to enhance the aeroelastic response through classical linear methods such as LQR or pole placement was usually adopted. In Singh and Wang (2002), an adaptive backstepping design technique was carried out to control the pitch angle with only output measurements. In Behal et al. (2006a), an adaptive control strategy was proposed using only the feedback for the pitching variable. Its performance toward suppressing flutter and LCOs as well as reducing the aeroelastic response in the subcritical flight speed regime was also demonstrated. Lee and Singh (2007) designed a robust control law for the global regulation of a 2 Degrees Of Freedom (DOF) aeroelastic system. The model had polynomial type structural nonlinearity and only the pitch angle was measured for feedback. It was also assumed that all the system parameters were unknown to the designer while the bounds of uncertainties were assumed to be known in the control design. Another robust control strategy for active flutter suppression of a nonlinear 2D wing-flap system was introduced in Zhang and Soffker (2009). An optimized state feedback robust stabilizer with a proportional-integral observer (PI-Observer) was designed where the PI-Observer was adopted to estimate both the system states and the bounds of the nonlinearities in the aeroelastic system. Based on the immersion and invariance approach, the adaptive control design problem for aeroelastic wing sections with structural nonlinearity was solved in Lee and Singh (2009).

Recently, a novel adaptive control scheme was presented (Behal et al., 2006b; Gujjula et al., 2005; Platanitis and Strganac, 2004; Rao et al., 2006; Reddy et al., 2007), which efficiently improved the performance through an extension to a wing section with two control surfaces. An adaptive fullstate feedback control law was provided in Platanitis and Strganac (2004). However, only an inversion of a nominal input gain matrix was used to decouple the control inputs without considering the uncertainty. In Gujjula et al. (2005), adaptive and Radial Basis Function (RBF) neural network controllers were provided in order to compensate for the system nonlinearity and compared via simulation. Note that a projection operator was adopted to assure that the input gain matrix estimate remains invertible. The adaptive control design problem for a non-linear $2 \mathrm{D}$ wing-flap system in supersonic/hypersonic flight speed was addressed in Rao et al. (2006). Instead of using projection introduced in Gujjula et al. (2005), an ST decomposition of the input gain matrix was used in Behal et al. (2006b) to design a singularity free controller which was applied on both leading (LECS) and trailing edge (TECS) control surfaces but requiring full-state feedback with a filtered tracking error. Here, $\mathrm{S}$ is a symmetric, positive matrix and $T$ is an upper triangular matrix with its diagonal elements belonging to the set $\{+1,-1\}$. In Reddy et al. (2007), an output feedback adaptive control algorithm was proposed by utilizing backstepping technique while an $S D U$ decomposition (symmetric-diagonal-upper triangular factorization) was applied on the input gain matrix to design a singular free controller. The aforementioned results under control with both TECS and LECS demonstrated better dynamic performance than using single control surface. In Wang et al. (2010), a modular output feedback controller was proposed to suppress aeroelastic vibrations on unmodeled nonlinear wing section subject to a variety of external disturbance. For details on backstepping design with $S D U$ factorization, the reader is referred to Tao (2003).

In this review paper, the latest development on the adaptive control design for subsonic and supersonic aeroelastic wing section model with one and two control surfaces are presented based on authors' previous work (Behal et al., 2006a, b; Rao et al., 2006; Reddy et al., 2007; Wang et al., 2010). In Behal et al. (2006a), control has been designed for a wing section model with only TECS and working at subsonic 
flight speed. For the supersonic/hypersonic wing section model, the reader is referred to Rao et al. (2006). Later, three control strategies (Behal et al., 2006b; Reddy et al., 2007; Wang et al., 2010) were given for the wing section model with both TECS and LECS at subsonic flight speed. Adaptive control, backstepping adaptive control, and neural network control have been implemented given different assumptions on this subsonic model while their performances were demonstrated in the following sections.

The rest of this paper is organized as follows. In Section 2, subsonic and supersonic system dynamics are introduced as well as gust and other disturbance model. In Section 3, several feedback and feedforward control designs are represented based on our previous paper. In Section 4, simulation results to confirm the performance of the proposed controllers are demonstrated. Conclusion is provided in Section 5.

\section{Mathematical Modeling}

\subsection{Aeroelastic governing equation}

A general 2-DOF subsonic pitch-plunge wing section model is considered in this paper. This aeroelastic governing equation is developed from previous models (Block and Strganac, 1998; Edwards et al., 1983; Platanitis and Strganac, 2004).

$\left[\begin{array}{cc}m_{T} & m_{w} x_{\alpha} b \\ m_{w} x_{\alpha} b & I_{\alpha}\end{array}\right]\left[\begin{array}{l}\ddot{h} \\ \ddot{\alpha}\end{array}\right]+\left[\begin{array}{cc}c_{h} & 0 \\ 0 & c_{\alpha}\end{array}\right]\left[\begin{array}{c}\dot{h} \\ \dot{\alpha}\end{array}\right]+\left[\begin{array}{cc}k_{h}(h) & 0 \\ 0 & k_{\alpha}(\alpha)\end{array}\right]\left[\begin{array}{l}h \\ \alpha\end{array}\right]=\left[\begin{array}{l}-L-L_{g} \\ M+M_{g}\end{array}\right] .(1)$

The definition of symbols used in the left hand side of the equation can be found in the nomenclature. The structural plunging and pitching stiffness polynomials $k_{h}(h)$ and $k_{\alpha}(\alpha)$ are of the following form

$$
k_{h}(h)=\sum_{i=1}^{3} k_{h, i} h^{i-1} \quad, \quad k_{\alpha}(\alpha)=\sum_{i=1}^{5} k_{\alpha, i} \alpha^{i-1}
$$

where $k_{h, i}$ and $k_{\alpha, i}$ are explicitly defined in Section

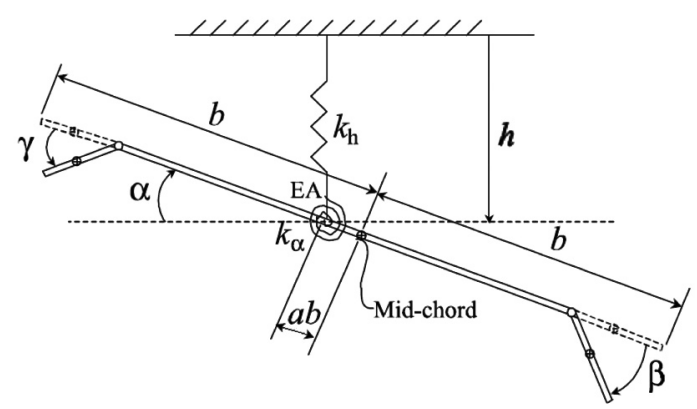

Fig. 1. Two-dimensional subsonic wing section aeroelastic model with both leading- and trailing-edge control surfaces. simulation. The aerodynamic lift $L$ and moment $M$ will be given in the following sections with respect to different aerodynamic models while $L_{g}$ and $M_{g}$ are the aerodynamic loads produced by an added gust load.

The wing section model in Eq. (1) with two control surfaces is given in Fig. 1, which can also be used to represent the model with only trailing edge control surface by assuming the leading edge control surface is rigidly mounted to the main surface $(\gamma \equiv 0 \mathrm{rad})$.

\subsection{Quasi-steady subsonic aerodynamic model}

The quasi-steady lift $L$ and moment $M$ can be given as

$L=\rho_{\infty} U_{\infty}^{2} b s C_{l \alpha}\left(\alpha+\frac{\dot{h}}{U_{\infty}}+\left(\frac{1}{2}-a\right) b \frac{\dot{\alpha}}{U_{\infty}}\right)+\rho_{\infty} U_{\infty}^{2} b s C_{l \beta} \beta+\rho_{\infty} U_{\infty}^{2} b s C_{l y} \gamma$,

$M=\rho_{\infty} U_{\infty}^{2} b^{2} s C_{m \alpha-e f f}\left(\alpha+\frac{\dot{h}}{U_{\infty}}+\left(\frac{1}{2}-a\right) b \frac{\dot{\alpha}}{U_{\infty}}\right)+\rho_{\infty} U_{\infty}^{2} b^{2} s C_{m \beta-e f f} \beta+\rho_{\infty} U_{\infty}^{2} b^{2} s C_{m y-e f f} \gamma$

where $C_{m \alpha-e f p} C_{m \beta-e f f}$ and $C_{m y-e f f}$ represent effective control moment coefficients derivatives and are defined as follows

$$
\begin{aligned}
& C_{m \alpha-e f f}=\left(\frac{1}{2}+a\right) C_{l \alpha}+2 C_{m \alpha}, \\
& C_{m \beta-e f f}=\left(\frac{1}{2}+a\right) C_{l \beta}+2 C_{m \beta}, \\
& C_{m y-e f f}=\left(\frac{1}{2}+a\right) C_{l \gamma}+2 C_{m \gamma} .
\end{aligned}
$$

\subsection{Unsteady subsonic aerodynamic model}

As extension to include an unsteady subsonic aerodynamic model for the 2-DOF aeroelastic wing section presented in Eq. (1), the unsteady aerodynamic lift $L$ and moment $M$ terms are given as

$$
L=\rho_{\infty} U_{\infty}^{2} b s C_{u L}(t)+P_{u}(t), M=\rho_{\infty} U_{\infty}^{2} b^{2} s C_{u M}(\tau)+Q_{u}(t)
$$

where $P_{u}(t)$ and $Q_{u}(t)$ are the externally applied force and moment, respectively. Here $P_{u}(t)$ and $Q_{u}(t)$ also include control surface loads as presented in Hariharan and Leishman (1996). For incompressible flow, $C_{u L}(t)$ and $C_{u M}(t)$ are the lift and pitching moment coefficients that can be expressed in the following forms

$$
\begin{aligned}
C_{u L}(t)= & \frac{\pi b}{U_{\infty}^{2}}\left(\ddot{h}-a b \ddot{\alpha}+U_{\infty} \dot{\alpha}\right)+\frac{2 \pi \varphi_{u}(t)}{U_{\infty}^{2}}\left[U_{\infty} \alpha(0)+\dot{h}(0)+\left(\frac{1}{2}-a\right) b \dot{\alpha}(0)\right] \\
& +\frac{2 \pi b}{U_{\infty}^{2}} \int_{0}^{t} \varphi_{u}(t-\sigma)\left[U_{\infty} \dot{\alpha}(\sigma)+\ddot{h}(\sigma)+\left(\frac{1}{2}-a\right) b \ddot{\alpha}(\sigma)\right] d \sigma, \\
C_{u M}(t)= & \frac{\pi \alpha b}{U_{\infty}^{2}}(\ddot{h}-\alpha b \ddot{\alpha})-\left(\frac{1}{2}-a\right) \frac{\pi b \dot{\alpha}}{U_{\infty}}-\frac{\pi b^{2} \ddot{\alpha}}{8 U_{\infty}^{2}} \\
& +\left(\frac{1}{2}+a\right) \frac{2 \pi \varphi_{u}(\tau)}{U_{\infty}}\left[U_{\infty} \alpha(0)+\dot{h}(0)+\left(\frac{1}{2}-a\right) b \dot{\alpha}(0)\right] \\
& +\frac{2 \pi b}{U_{\infty}^{2}}\left(\frac{1}{2}+a\right) \int_{0}^{\tau} \varphi_{u}(\tau-\sigma)\left[U_{\infty} \dot{\alpha}(\sigma)+\ddot{h}(\sigma)+\left(\frac{1}{2}-a\right) \ddot{\alpha}(\sigma)\right] d \sigma
\end{aligned}
$$

where the Wagner function $\varphi_{u}(\tau)$ can be given as

$$
\varphi_{u}(\tau)=1-\psi_{u 1} e^{-\varepsilon_{u 1} \tau}-\psi_{u 2} e^{-\varepsilon_{u 2} \tau}
$$


where $\tau=U_{\infty} t / b$ is dimensionless time variable, $\psi_{u l}, \psi_{u 2}, \varepsilon_{u l}$, and $\varepsilon_{u 2}$ are the Wagner's constants. Note that Eqs. (5) and (6) have been rewritten from a classical model, presented for example in Fung (2002), as to provide a unified framework consistent with the aeroelastic governing equations in Eq. (1) and the other aerodynamic load equations provided in the quasi-steady and supersonic aerodynamic modeling formulation. As this review puts an emphasis on adaptive and robust control strategy development, the discussions presented in the next sections are limited to quasi-steady aerodynamic formulations. However, following Ko et al. (1997), the control design for the model with extension to include unsteady aerodynamics can also be implemented straightforwardly based on the strategies (Leishman, 1988, 1994; Marzocca et al., 2001, 2002a, b; Qin et al., 2002).

\subsection{Supersonic aerodynamic model and piston theory aerodynamics}

For the aeroelastic wing section model at supersonic flight speed regime with plunging and pitching degrees of freedom, consistent with piston theory aerodynamics (PTA), the aerodynamic lift $\mathrm{L}$ and moment $M$ can be expressed in a form to account for flap deflection as

$$
\begin{aligned}
L= & \frac{b U_{\infty} \rho_{\infty}}{3 \bar{M}} \lambda\left\{12 U_{\infty} \alpha(t)+\bar{M}^{2} U_{\infty}(1+\bar{\gamma}) \lambda^{2} \alpha^{3}\right. \\
& \left.-3\left[2 U_{\infty}\left(-2+x_{1}\right) \beta(t)+4\left(-b+x_{E A}\right) \dot{\alpha}-b\left(-2+x_{1}\right)^{2} \dot{\beta}(t)-4 \dot{h}\right]\right\}, \\
M= & -\frac{b U_{\infty} \rho_{\infty}}{3 \bar{M}}\left\{12 U_{\infty}\left(b-x_{E A}\right) \alpha(t)+\bar{M}^{2} U_{\infty}\left(b-x_{E A}\right)(1+\bar{\gamma}) \lambda^{2} \alpha^{3}\right. \\
& -3 b U_{\infty}\left(-2+x_{1}\right)\left(2-2 x_{0}+x_{1}\right) \beta(t)+b U_{\infty}\left(-2+x_{1}\right)^{2}\left(4-3 x_{0}+x_{1}\right) \\
& \left.+4\left[3\left(b-x_{E A}\right) \dot{h}+\left(4 b^{2}-6 b x_{E A}+3 x_{E A}^{2}\right) \dot{\alpha}\right]\right\}
\end{aligned}
$$

where $\bar{M}=U_{\infty} / a_{\infty}$ is the undisturbed flight Mach number and $\mathrm{a}_{\infty}$ is the speed of the sound. $\lambda=\bar{M} / \sqrt{\bar{M}^{2}-1}$ enables one to extend the validity of the PTA to the entire low supersonichypersonic flight speed regime. $\bar{\gamma}$ is the isentropic gas coefficient, ( $\bar{\gamma}=1.4$ for dry air). Also note that $x_{E A}=b x_{0}$ while $x_{0}$ and $x_{1}$ represent the dimensionless locations of the elastic axis and of the torsional spring of the flap from the leadingedge respectively.

\subsection{Gust and other disturbance}

Based on Marzocca et al. (2001), the aerodynamic loads due to the bounded external disturbance can be expressed as follows

$$
\begin{aligned}
& L_{g}=\rho U_{\infty}^{2} b s C_{l \alpha} w_{G}(\tau) / U_{\infty}=\rho U_{\infty} b s C_{l \alpha} w_{G}(\tau), \\
& M_{g}=\left(\frac{1}{2}-a\right) b L_{g}
\end{aligned}
$$

where $W_{G}(\tau)$ represents three types of velocity distribution functions for the external disturbances considered in this paper. The first type of external disturbance can be modeled as a triangular gust with the following velocity distribution function

$w_{G}(\tau)=2 w_{0} \frac{\tau}{\tau_{G}}\left(H(\tau)-H\left(\tau-\frac{\tau_{G}}{2}\right)\right)-2 w_{0}\left(\frac{\tau}{\tau_{G}}-1\right)\left(H\left(\tau-\tau_{G}\right)-H\left(\tau-\frac{\tau_{G}}{2}\right)\right)$

where $H(\cdot)$ is a unit step function and $\tau_{G}=U_{\infty} t_{G} / b$. Note that $t_{G}$ and $w_{0}$ are determined based on the different simulation setting. The second type of external disturbance, which is sustained beyond the transient response time of the closedloop aeroelastic system, can be represented in the form of graded gust, whose velocity distribution $W_{G}(\tau)$ can be written as follows

$$
w_{G}(\tau)=H(\tau) w_{0}\left(1-e^{-0.75 \tau}\right)
$$

As we mentioned before, $w_{0}$ need to be chosen according to the simulation setting. The last type of external disturbance tested in this paper is given in the form of sinusoidal gust with the following velocity distribution function

$$
w_{G}(\tau)=H(\tau) w_{0} \sin (\omega \tau)
$$

where $\omega$ and $w_{0}$ are selected case by case. Thus, above three types of disturbance profiles examine the response of the aeroelastic system to ephemeral disturbances, steady sustained disturbances, and time-varying sustained disturbances. Here we note that the triangular gust examined in this paper is quite similar to the classical 1-cosine gust-type function since both of them can be classified as ephemeral disturbances. Meanwhile, our control algorithm is also verified under a more challenging continuous sinusoidal disturbance.

\section{State Feedback and Output Feedback Control Development Nonlinear}

\subsection{Adaptive control for subsonic airfoils}

Given a subsonic quasi-steady aerodynamic model based on Eqs. (1) and (3), where only TECS is considered $\left(\gamma(\mathrm{t}) \equiv 0, L_{\mathrm{g}}\right.$ $\equiv 0$, and $M_{g} \equiv 0$ ), the governing equations for the aeroelastic model can be expressed in the following convenient statespace form

$$
\begin{aligned}
& \dot{\mathbf{x}}_{1}=\mathbf{A}_{1} \mathbf{x}_{1}+\Phi_{1}\left(y_{1}\right)+\mathbf{B}_{1} \bar{\beta}, \\
& y_{1}=\mathbf{C}_{1}^{T} \mathbf{x}_{1}=\left[\begin{array}{llll}
1 & 0 & 0 & 0
\end{array}\right] \mathbf{x}_{1} .
\end{aligned}
$$

Herein, $\bar{\beta}=U_{\infty}^{2} \beta \in \mathrm{R}^{1}$ is an auxiliary control input, $\mathbf{x}_{1}(t) \in \mathrm{R}^{4}$ 
is a new vector of system states, $y_{1} \in \mathrm{R}^{1}$ is the output variable, $\mathbf{A}_{1} \in \mathrm{R}^{4 \times 4}$ and $\mathbf{B}_{1} \in \mathrm{R}^{4}$ are explicitly defined as follows

$$
\mathbf{A}_{1}=\left[\begin{array}{llll}
0 & 1 & 0 & 0 \\
0 & 0 & 1 & 0 \\
0 & 0 & 0 & 1 \\
0 & 0 & 0 & 0
\end{array}\right], \quad \mathbf{B}_{1}=\left[\begin{array}{c}
0 \\
\theta_{1,2} \\
\theta_{1,3} \\
\theta_{1,4}
\end{array}\right]
$$

where $\theta_{1, i} \forall i=2,3,4$ are constants. $\boldsymbol{\Phi}_{1}\left(y_{1}\right) \in \mathrm{R}^{4}$ in Eq. (14) is a smooth vector field that can be linearly parameterized as follows

$$
\Phi_{1}\left(y_{1}\right)=\mathbf{W}_{1}\left(y_{1}\right) \gamma_{1}=\sum_{j=1}^{p_{1,0}} \gamma_{1, j} \mathbf{W}_{1, j}\left(y_{1}\right)
$$

where $\gamma_{1} \in \mathrm{R}^{p 1,0}$ is a vector of constant unknowns while $\gamma_{1, \mathrm{j}}$ denotes the jth element in $\gamma_{1}, \mathbf{W}_{1}(\cdot) \in \mathrm{R}^{4 \times p 1,0}$ is a measurable, nonlinear regression matrix while the notation $\mathbf{W}_{1, j}(\cdot) \in \mathrm{R}^{4}$ $\forall j=1, \ldots, p_{1,0}$ denotes the $\mathrm{jth}$ column of the regression matrix $\mathbf{W}_{1}(\cdot)$. The expressions of $\gamma_{1}$ depends on the choice of pitch spring nonlinearity polynomial $k_{\alpha}(\alpha)$, see e.g. (Behal et al., 2006a). Also note that the proposed control strategy in this section is based on the assumption that the system of Eq. (14) is minimum phase.

The control algorithm designed in this section aims to drive the pitch angle $\alpha$ to zero by using $y_{1}=\alpha$ as the only measurement available. Thus, compensation is needed for the remaining states via use of state estimators. The pitch angle setpoint error $e_{1,1}(t) \in \mathrm{R}^{1}$ and state estimation error $\tilde{\boldsymbol{x}}_{1}(t) \in \mathrm{R}^{4}$ are defined as follows

$$
e_{1,1}=y_{1}-y_{1, d} \quad, \quad \tilde{\mathbf{x}}_{1} \equiv \mathbf{x}_{1}-\hat{\mathbf{x}}_{1}
$$

where $\hat{\mathbf{x}}_{1}(t) \in \mathrm{R}^{4}$ is a state estimate that is yet to be designed. $y_{1, d} \in \mathrm{R}^{1}$ is the reference output. Based on the assumption that the model parameters are unknown, the parameter estimation error signals $\tilde{\boldsymbol{\sigma}}_{1,0}(t) \in \mathrm{R}^{p 1,1}$ and $\tilde{\boldsymbol{\sigma}}_{1,1}(t) \in \mathrm{R}^{p 1,2}$ can be defined as follows

$$
\tilde{\sigma}_{1,0}=\sigma_{1,0}-\hat{\sigma}_{1,0} \quad, \quad \tilde{\sigma}_{1,1}=\sigma_{1,1}-\hat{\sigma}_{1,1}
$$

where $\boldsymbol{\sigma}_{1,0} \in \mathrm{R}^{p 1,1}$ and $\boldsymbol{\sigma}_{1,1} \in \mathrm{R}^{p 1,2}$ are unknown constant vectors. $\hat{\boldsymbol{\sigma}}_{1,0}(t) \in \mathrm{R}^{p 1,1}$ and $\hat{\boldsymbol{\sigma}}_{1,1}(t) \in \mathrm{R}^{p 1,2}$ are the dynamic estimates for $\boldsymbol{\sigma}_{1,0}$ and $\boldsymbol{\sigma}_{1,1}$, respectively, and will be designed later. Following the work of Zeng and Singh (1998), a bank of filters can be designed as

$$
\begin{aligned}
& \dot{\zeta}_{1,0}=\mathbf{A}_{1} \zeta_{1,0}+\mathbf{K}_{1} \mathbf{C}_{1}^{T}\left(\mathbf{x}_{1}-\zeta_{1,0}\right), \\
& \dot{\zeta}_{1, j}=\mathbf{A}_{1,0} \zeta_{1, j}+\mathbf{W}_{1, j}\left(y_{1}\right), \quad \forall j=1 \ldots p_{1,0}, \\
& \dot{v}_{1, i}=\mathbf{A}_{1,0} \mathbf{v}_{1, i}+\lambda_{1, i} \bar{\beta}, \quad \forall i=2,3,4,
\end{aligned}
$$

where $\lambda_{1, i} \forall i=2,3,4$ denotes the standard basis vectors and $\mathbf{A}_{1,0}=\mathbf{A}_{1}-\mathbf{K}_{1} \mathbf{C}_{1}^{T}$ is Hurwitz by choosing gain vector $\mathbf{K}_{1}$ properly. $\zeta_{1, j} \in \mathrm{R}^{4} \forall j=0, \ldots, p_{1,0}$ and $v_{1, i} \in \mathrm{R}^{4} \forall i=2,3,4$ are filtered variables. Given Eq. (19), an immeasurable state estimate $\hat{\mathbf{x}}_{1}(t)$ can be patched together as follows

$$
\hat{\mathbf{x}}_{1}=\zeta_{1,0}+\sum_{j=1}^{p_{1,0}} \gamma_{1, j} \zeta_{1, j}+\sum_{j=2}^{4} \theta_{1, j} \boldsymbol{v}_{1, j}
$$

By taking the derivative of $\tilde{\mathbf{x}}_{1}$ given in Eq. (17) and substituting for the dynamics of Eqs. (14) and (20), the following exponentially stable estimation error system can be obtained

$$
\dot{\tilde{\mathbf{x}}}_{1}=\mathbf{A}_{1,0} \tilde{\mathbf{x}}_{1}
$$

Similar to Zeng and Singh (1998), an auxiliary error signal $e_{1,2}(t) \in \mathrm{R}^{1}$ is defined as $e_{1,2}=v_{1,2,2}-v_{1,2,2 d}$ where $v_{1,2, \mathrm{i}}$ denotes the ith element in $v_{1,2}$ given in Eq. (19). Also note that $v_{1,2,2 \mathrm{~d}}$ can be given as follows

$$
v_{1,2,2 d}=-\omega_{1,1}^{T} \hat{\sigma}_{1,0}
$$

where $\hat{\boldsymbol{\sigma}}_{1,0}$ and $\boldsymbol{\omega}_{1,1}(t)$ denote, respectively, a vector of unknown constants and a measurable regression vector, explicitly defined in Behal et al. (2006a). The dynamic adaptive update law for $\hat{\boldsymbol{\sigma}}_{1,0}$ is selected as follows

$$
\dot{\hat{\sigma}}_{1,0}=\operatorname{sign}\left(\theta_{1,2}\right) \omega_{1,1} e_{1,1} \text {. }
$$

The next step involves the design of the actual control input $\bar{\beta}(t)$ which is reachable through backstepping

$$
\bar{\beta}(t)=-c_{22} e_{1,2}-d_{22}\left(\frac{\partial v_{1,2,2 d}}{\partial y_{1}}\right)^{2} e_{1,2}-v_{1,2,3}+k_{1,2} v_{1,2,1}+\dot{v}_{1,2,2 d m}-\omega_{1,2}^{T} \hat{\sigma}_{1,1} .
$$

In the above equation, $\dot{v}_{1,2,2 d m}(t) \in \mathrm{R}^{1}$ denotes the measurable component that is matched with the control input $\bar{\beta}(t)$ and can be done away via direct cancellation. $v_{1,2, \mathrm{i}}$ denotes the ith element in $\boldsymbol{v}_{1,2}$ given in Eq. (19). The estimates $\hat{\boldsymbol{\sigma}}_{1,1}$ can obtained via the following adaptive update law

$$
\dot{\hat{\sigma}}_{1,1}=\omega_{1,2} e_{1,2} .
$$

For explicit expressions of $\dot{v}_{1,2,2 d m}(t), \omega_{1,2}, c_{22}, d_{22}$, and $k_{1,2}$, the reader is referred to Behal et al. (2006a). A Lyapunov stability analysis shows that $\lim e_{1,1}(t), e_{1,2}(t)=0$ and the estimation error $\tilde{\mathbf{x}}_{1}(t)$ is exponentially regulated to the origin based on Eq. (21).

To model the control surface dynamics associated with the quasi-steady aerodynamic equations described in Eqs. (1) and (3), the method in Block and Strganac (1998) is utilized and a second-order system is proposed as follows 


$$
\ddot{\beta}+p_{1,1} \dot{\beta}+p_{1,2} \beta=u_{1} \quad, \quad p_{1,2} \neq 0
$$

Two different strategies are proposed to include the effect of the dynamics of the flap. In the first strategy, $p_{1,1}$ and $p_{1,2}$ are chosen such that the dynamics of Eq. (26) are faster than the dynamics of Eq. (1). Then, the control signal $\beta(t)$ given in Eq. (24) can be treated as a desired flap deflection $\beta_{\mathrm{d}}(t)$ and the control input $u_{1}(t)$ can be simply designed as

$$
u_{1}(t)=\ddot{\beta}_{d}+p_{1,1} \dot{\beta}_{d}+p_{1,2} \beta_{d} .
$$

After defining $\varepsilon_{1}=\beta-\beta_{d}$, the closed-loop system for Eq. (26) becomes

$$
\ddot{\varepsilon}_{1}+p_{1,1} \dot{\varepsilon}_{1}+p_{1,2} \varepsilon_{1}=0
$$

which is an exponentially stable system. Thus, $\beta(t)$ converges to $\beta_{d}(t)$ exponentially. However, when the dynamics of actuator is slow, the interconnection effect between the dynamics of Eqs. (1) and (26) needs to be considered. Given $v_{\beta}(t)=\dot{\beta}(t)$, an auxiliary design variable is defined as $\eta_{v}(t)=v_{\beta}-v_{\beta d} \in \mathrm{R}^{1}$ where $v_{\beta d}(t)$ is a virtual control input that can be given as

$$
v_{\beta d}=\dot{\beta}_{d}-U_{\infty}^{2} e_{1,2}+k_{\varepsilon} \varepsilon_{1}
$$

where $k_{\varepsilon}$ is a positive constant. Based on above results and the backstepping technique, the control input $u_{1}$ can be designed as follows

$$
u_{1}(t)=p_{2,1}^{-1}\left[\dot{v}_{\beta d}+p_{1,1} \dot{\beta}+p_{1,2} \beta-\varepsilon_{1}-k_{\eta} \eta_{v}\right]
$$

where $k_{\eta}$ is a positive constant. A signal chasing argument and stability analysis in Behal et al. (2006a) show that lim $e_{1,1}(t), e_{1,2}(t), \tilde{\mathbf{x}}_{1}(t)=0$.

\subsection{Nonlinear adaptive control for supersonic airfoil}

After denoting the nondimensional time $\tau=U_{\infty} t / b$ and nondimensional plunging displacement $\xi=h / b$ variables, one can transform the dimensional Eqs. (1), (8), and (9) into the nondimensional form Rao et al. (2006), which can be further rewritten into the following state space form in order to facilitate output feedback control design

$$
\begin{aligned}
& \dot{\mathbf{x}}_{2}=\mathbf{A}_{2} \mathbf{x}_{2}+\left[\Phi_{2}\left(y_{2}\right)+\mathbf{B}_{2} \bar{\beta}\right], \\
& y_{2}=\mathbf{C}_{2}^{T} \mathbf{x}_{2}=\left[\begin{array}{llll}
1 & 0 & 0 & 0
\end{array}\right] \mathbf{x}_{2}
\end{aligned}
$$

where $\bar{\beta}=U_{\infty}^{2} \beta \in \mathrm{R}^{1}$ is an auxiliary control input, $\mathbf{x}_{2}(t)=\left[x_{2,1}(\mathrm{t})\right.$ $\left.x_{2,2}(t) \quad x_{2,3}(t) \quad x_{2,4}(t)\right]^{\mathrm{T}} \in \mathrm{R}^{4}$ is a new vector of system states, $y_{2}$ is the output variable. $\mathbf{A}_{2}$ is the same as $\mathbf{A}_{1}$ while $\mathbf{B}_{2} \in \mathrm{R}^{4}$ is defined as

$$
\mathbf{B}_{2}=\left[\begin{array}{llll}
\theta_{2,1}, & \theta_{2,2}, & \theta_{2,3}, & \theta_{2,4}
\end{array}\right]^{T}
$$

where $\theta_{2, i} \in \mathrm{R}_{1} \forall i=1,2,3,4$ are constants that are explicitly defined in Rao et al. (2006). Note that $\theta_{2, i} \neq 0$ leads to a relative degree one system. In (31) above, $\boldsymbol{\Phi}_{2}\left(y_{2}\right) \in \mathrm{R}_{4}$ can be linearly parameterized as follows

$$
\Phi_{2}\left(y_{2}\right)=\mathbf{W}_{2,1}\left(y_{2}\right) \bar{\gamma}_{2}=\sum_{j=1}^{p_{2}} \bar{\gamma}_{2, j} \mathbf{W}_{2,1, j}\left(y_{2}\right)
$$

where $\bar{\gamma}_{2} \in \mathrm{R}^{p 2}$ is a vector of constant unknowns while $\bar{\gamma}_{2, j}$ denotes the $j$ th element in $\bar{\gamma}_{2}, \mathbf{W}_{2,1}\left(y_{2}\right) \in \mathrm{R}^{4 \times p 2}$ is a measurable, non-linear regression matrix while the notation $\mathbf{W}_{2,1}\left(y_{2}\right) \in \mathbf{R}^{4}$ denotes the jth column of the regression matrix $\mathbf{W}_{2,1}\left(y_{2}\right) \forall j=1$, $\ldots, p_{2}$. Explicit expressions for $\bar{\gamma}_{2}$ in terms of model parameters are provided in Rao et al. (2006).

Based on the known structure of the aeroelastic model, our goal in this section is to design a control strategy to drive the pitch angle $\alpha$ to zero and adaptively compensating for the parametric uncertainties as well as the nonlinearity. Assuming that the only measurement available is the output variable $y_{2}=\alpha$ while the remaining states needs to be estimated through the state estimators, a state estimation error $\tilde{\mathbf{x}}_{2}(t) \in \mathrm{R}^{4}$ can be designed as follows

$$
\tilde{\mathbf{x}}_{2}=\mathbf{x}_{2}-\hat{\mathbf{x}}_{2}
$$

where $\mathbf{x}_{2}(t)$ is a state vector previously defined in Eq. (31) while $\tilde{\mathbf{x}}_{2}(t) \in \mathrm{R}^{4}$ is an estimator variable that is yet to be designed. The parameter estimation error signal $\tilde{\chi}_{2}(t) \in \mathrm{R}^{r}$ is represented as

$$
\tilde{\chi}_{2}=\chi_{2}-\hat{\chi}_{2}
$$

where $\chi_{2}(t) \in \mathrm{R}^{r}$ is an unknown constant parameter vector while $\hat{\chi}_{2}(t) \in \mathrm{R}^{\mathrm{r}}$ is its corresponding dynamic estimate. The bracketed term in the first Eq. (31) can be linearly parameterized as

$$
\mathbf{W}_{2,2}\left(y_{2}, \bar{\beta}\right) \sigma_{2}=\sum_{j=1}^{q_{2}} \sigma_{2, j} \mathbf{W}_{2,2, j}\left(y_{2}, \bar{\beta}\right)=\Phi_{2}\left(y_{2}\right)+\mathbf{B}_{2} \bar{\beta}
$$

where $\boldsymbol{\sigma}_{2} \in \mathrm{R}^{q 2}$ is a vector of constant unknowns while $\sigma_{2, j}$ denotes the jth element in $\boldsymbol{\sigma}_{2}, \mathbf{W}_{2,2}\left(y_{2}, \bar{\beta}\right) \in \mathrm{R}^{4 \times q_{2}}$ is a measurable, non-linear regression matrix while the notation $\mathbf{W}_{2,2, j}(\cdot) \in \mathrm{R}^{4}$ denotes the $j$ th column of the regression matrix $\mathbf{W}_{2,2}(\cdot) \forall j=1, \ldots, q_{2}$. Next, an immeasurable estimation variable $\hat{x}_{2}(t) \in \mathrm{R}^{4}$ is defined as follows

$$
\hat{\mathbf{x}}_{2}=\zeta_{2,0}+\sum_{j=1}^{q_{2}} \sigma_{2, j} \zeta_{2, j}, \quad \forall j=1, \cdots, q_{2}
$$


where $\zeta_{2,0} \in \mathrm{R}^{4}$ and $\zeta_{2, j} \in \mathrm{R}^{4} \forall j=1, \ldots, q_{2}$ are filter variables such that (Krstic et al., 1995)

$$
\begin{aligned}
& \dot{\zeta}_{2,0}=\mathbf{A}_{2,0} \zeta_{2,0}+\mathbf{K}_{2} \mathbf{C}_{2}^{T} \mathbf{x}_{2}, \\
& \dot{\zeta}_{2, j}=\mathbf{A}_{2,0} \zeta_{2, j}+\mathbf{W}_{2,2, j}\left(y_{2}, \bar{\beta}\right) \quad \forall j=1, \cdots, q_{2}
\end{aligned}
$$

where $\mathbf{K}_{2} \in \mathrm{R}^{4}$ is a gain vector chosen so that $\mathbf{A}_{2,0}=\mathbf{A}_{2}-\mathbf{K}_{2} \mathbf{C}_{2}^{T}$ is Hurwitz. Given that $\mathbf{A}_{2}, \mathbf{C}_{2}^{T} \mathbf{x}_{2}=y_{2}$, and $\mathbf{W}_{2,2, j}\left(y_{2}, \bar{\beta}\right)$ are known, one can see that the design of Eq. (38) is readily implementable. By taking the derivative of Eq. (34) and substituting for the dynamics of Eqs. (31) and (37), one can obtain the following exponentially stable estimation error

$$
\dot{\tilde{\mathbf{x}}}_{2}=\mathbf{A}_{2} \tilde{\mathbf{x}}_{2} .
$$

Although the state estimate ofEq. (37) is unmeasurable, the worth of the above scheme lies in the linear parameterizability of the right hand side of the expression of Eq. (37) as well as the exponential stability of the estimation error. Based on the structure of Eq. (31), the control input $\bar{\beta}(t)$ can be designed as

$$
\bar{\beta}=-\mathbf{W}_{2,3}\left(y_{2}, \zeta_{2,0,2}, \zeta_{2, j, 2}\right) \hat{\chi}_{2}
$$

where $\zeta_{2, j, i}$ denotes the ith element in $\zeta_{2, j} \cdot \mathbf{W}_{2,3}^{T}\left(y_{2}, \zeta_{2,0,2}\right.$, $\left.\zeta_{2, j, 2}\right) \in \mathrm{R}^{r}$ is a measurable regression vector such that

$$
\mathbf{W}_{2,3}\left(y_{2}, \zeta_{2,0,2}, \zeta_{2, j, 2}\right) \hat{\chi}_{2}=\theta_{2,1}^{-1}\left[\theta_{2,1} k_{p} x_{2,1}+\zeta_{2,0,2}+\sum_{j=1}^{q_{2}} \zeta_{2, j, 2} \sigma_{2, j}+\Phi_{2,1}\left(y_{2}\right)\right] .
$$

where the control gain $k_{\mathrm{p}}>0$ as reported in Rao et al. (2006), $\Phi_{2, j}\left(y_{2}\right)$ represents the jth element in $\boldsymbol{\Phi}_{2}\left(y_{2}\right), x_{2,1}=y$, and $\zeta_{2,0, i}$ denotes the ith element in $\zeta_{2,0}$. The parameter estimate vector $\hat{x}_{2}(t)$ is computed online as follows

$$
\dot{\hat{\chi}}_{2}=\operatorname{sign}\left(k_{2}\right) \mathbf{W}_{2,3}^{T}\left(y_{2}, \zeta_{2,0,2}, \zeta_{2, j, 2}\right) x_{2,1}
$$

where the definition of $k_{2}$ can be found in Rao et al. (2006). Given all the preceding facts, it is straightforward to prove that $\lim _{t \rightarrow \infty} x_{2,1}(t)=y=0$ and the estimation error $\tilde{\mathbf{x}}_{2}(t)$ is exponentially regulated to the origin, as shown in the stability analysis in Rao et al. (2006).

\subsection{Adaptive control for a nonlinear wing section with multiple flaps}

For the wing section model described by Eqs. (1) and (3) with both TECS and LECS available, an input-output form can be derived for the system as follows

$$
\ddot{\mathbf{y}}_{3}=\mathbf{f}_{3 \mu}\left(\alpha, \dot{\alpha}, h, \dot{h}, \theta_{3,1}\right)+\mathbf{G}_{3 \mu}\left(\theta_{3,2}\right) \mathbf{u}
$$

where $\mathbf{y}_{3}(t) \stackrel{\Delta}{=}[\alpha(t), h(t)]^{T} \in \mathrm{R}^{2}$ denotes the output vector, $\mathbf{u}(t) \stackrel{\Delta}{=} U_{\infty}^{2}[\beta(t), \gamma(t)]^{T} \in \mathrm{R}^{2}$ denotes the control input vector.
$\mathbf{G}_{3 \mu} \in \mathrm{R}^{2 \times 2}$ is a constant non-singular (but sign-indefinite), non-symmetric, gain matrix. In Eq. (43), $\theta_{3,1} \in \mathrm{R}^{p_{3,1}}$ and $\theta_{3,2} \in \mathrm{R}^{p_{3,2}}$ denote unknown model parameter vectors. Since both the leading principal minors $g_{3,11}$ and $\Delta 3=\operatorname{det}\left(\mathbf{G}_{3 \mu}\right)$ of the matrix $\mathbf{G}_{3 \mu}$ are non-zero, a matrix decomposition introduced in (Morse, 1993) is used to obtain $\mathbf{G}_{3 \mu}=\mathbf{S}_{3} \mathbf{T}_{3}$ where $\mathbf{S}_{3}$ is a symmetric, positive-definite matrix while $\mathbf{T}_{3}$ is an upper triangular matrix with its diagonal elements belonging to the set $\{+1,-1\}$. Based on above matrix decomposition, Eq. (43) can be rewritten as follows

$$
\mathbf{M}_{3}\left(\theta_{3,2}\right) \frac{d^{2} \mathbf{y}_{3}}{d t}=\mathbf{f}_{3}\left(\alpha, \dot{\alpha}, h, \dot{h}, \theta_{3,1}, \theta_{3,2}\right)+\operatorname{det}\left(\mathbf{S}_{3}\left(\theta_{3,2}\right)\right) \mathbf{T}_{3}\left(\theta_{3,2}\right) \mathbf{u}
$$

where $\mathbf{M}_{3}(\cdot)=\operatorname{adj}\left(\mathbf{S}_{3}(\cdot)\right) \in \mathrm{R}^{2 \times 2}$ is symmetric p.d., while $\mathbf{f}_{3}(\cdot)=\mathbf{M}_{3}(\cdot) \mathbf{f}_{3 \mu}(\cdot) \in \mathrm{R}^{2}$ is an auxiliary vector. The control objective in this section is to suppress plunging and pitching oscillations (possibly along desired trajectories generated by a reference model) in the presence of parametric uncertainties $\theta_{3,1}, \theta_{3,2}$. Herein, assuming that $\alpha(t), \dot{\alpha}(t), h(t)$, and $\dot{h}(t)$ are measurable, a tracking error $\mathbf{e}_{3}(t) \in \mathrm{R}^{2}$ is defined as

$$
\mathbf{e}_{3}=\mathbf{y}_{3, d}-\mathbf{y}_{3}
$$

where $\mathbf{y}_{3, d}(t) \in \mathrm{R}^{2}$ is a reference trajectory. In order to achieve the desired objective, a sliding surface $\mathbf{r}_{3}(t) \in \mathrm{R}^{2}$ is assumed as

$$
\mathbf{r}_{3}(t) \stackrel{\Delta}{=} \dot{\mathbf{e}}_{3}+\Lambda_{3,1} \mathbf{e}_{3}
$$

where $\Lambda_{3,1} \in \mathrm{R}^{2 \times 2}$ is a constant, positive definite matrix chosen such that the resulting characteristic polynomial is Hurwitz. Motivated by the subsequent stability analysis, a parameter estimation error is also defined in the following form

$$
\tilde{\theta}_{3}=\theta_{3}-\hat{\theta}_{3} \quad, \quad \tilde{\theta}_{3, s}(\mathbf{t})=\theta_{3, s}-\hat{\theta}_{3, s}
$$

where $\boldsymbol{\theta}_{3} \in \mathrm{R}^{P 3,3}$ and $\boldsymbol{\theta}_{3, s} \in \mathrm{R}^{P 3,4}$ contain the unknown system parameters, $\hat{\boldsymbol{\theta}}_{3}(t) \in \mathrm{R}^{P 3,3}$ and $\hat{\boldsymbol{\theta}}_{3, s}(t) \in \mathrm{R}^{P 3,4}$ denote their yet to be designed estimates, respectively. Based on above analysis, the adaptive control law $\mathbf{u}$ can be designed as follows

$$
\mathbf{u}=\mathbf{D}_{3,2}\left(\mathbf{y}_{3, s} \hat{\theta}_{3, s}\right)^{-1}\left(\mathbf{Y}_{3} \hat{\theta}_{3}+\mathbf{K}_{3} \mathbf{r}_{3}\right)
$$

where $\mathbf{K}_{3} \in \mathrm{R}^{2 \times 2}$ is a constant, positive definite gain matrix. $\mathbf{D}_{3,2}=\operatorname{diag}\left\{\operatorname{sign}\left(g_{3,11}\right), \operatorname{sign}\left(g_{3,11}\right) \operatorname{sign}(\Delta)\right\}$ is a diagonal matrix which is assumed to be known. The estimates $\hat{\boldsymbol{\theta}}_{3}$ and $\hat{\boldsymbol{\theta}}_{3, s}(t)$ are dynamically generated as follows

$$
\dot{\hat{\theta}}_{3}=\Gamma_{3} \mathbf{Y}_{3}^{T} \mathbf{r}_{3},
$$




$$
\dot{\hat{\theta}}_{3, s}=\operatorname{Proj}\left\{\Gamma_{3, s} \mu_{3}\right\}, \quad \mu_{3}=-\mathbf{y}_{3, s}^{T}\left(\mathbf{Y}_{3} \hat{\theta}_{3}+\mathbf{K}_{3} \mathbf{r}_{3}\right)^{T}\left(\mathbf{y}_{3, s} \hat{\theta}_{3, s}\right)^{-1} \mathbf{r}_{3},
$$

where $\boldsymbol{\Gamma}_{3} \in \mathrm{R}^{p_{3,3} \times p_{3,3},}, \quad \boldsymbol{\Gamma}_{3, s} \in \mathrm{R}^{p_{3,4} \times p_{3,4}}$ are constant, positive definite gain matrices while $\operatorname{Proj}\{\bullet\}$ is a standard parameter projection operator used to ensure that $\mathbf{y}_{3, s} \hat{\boldsymbol{\theta}}_{3, s}>0$ for all time (Behal et al., 2006b). $\mathbf{Y}_{3}(\cdot) \in \mathrm{R}^{2 \times p_{3,3}}$ and $\mathbf{y}_{3, s}(\cdot) \in \mathrm{R}^{I \times p_{3,4}}$ are measurable regressors that are explicitly defined as

$$
\begin{aligned}
& \mathbf{Y}_{3}(\cdot) \theta_{3}=\left[\mathbf{M}_{3}\left(\ddot{\mathbf{y}}_{d}+\Lambda_{3,1} \dot{\mathbf{e}}_{3}\right)-\mathbf{f}_{3}-\operatorname{det}\left(\mathbf{S}_{3}\right) \overline{\mathbf{T}}_{3} \mathbf{u}+\frac{1}{2} \dot{\mathbf{M}}_{3} \mathbf{r}_{3}\right], \\
& \mathbf{y}_{3, s}(\cdot) \theta_{3, s}=\operatorname{det}\left(\mathbf{S}_{3}\right)
\end{aligned}
$$

where the strictly upper triangular matrix $\overline{\mathbf{T}}_{3}\left(\boldsymbol{\theta}_{3,2}\right)$ can be d

$$
\overline{\mathbf{T}}_{3}\left(\theta_{3,2}\right)=\mathbf{T}_{3}\left(\theta_{3,2}\right)-\mathbf{D}_{3,2} .
$$

Notice that this strict upper triangularity allows one to design control in a hierarchical fashion without the possibility of any static or algebraic loops. Stability analysis in Behal et al. (2006b) shows that $\lim _{t \rightarrow \infty} \mathbf{r}_{2}(t)=0$.

\subsection{MIMO adaptive output control for aeroelastic vibration suppression}

First of all, the governing equations in Eqs. (1) and (3) can be transformed into the following form in order to facilitate output feedback control design

$$
\begin{aligned}
& \dot{\mathbf{x}}_{4}=\mathbf{A}_{4} \mathbf{x}_{4}+\overline{\mathbf{W}}_{4}\left(\mathbf{y}_{4}, \varphi_{4}\right)+\left[\begin{array}{c}
\mathbf{0}_{2} \\
\mathbf{G}_{4, \mu}
\end{array}\right] \mathbf{u}, \\
& \mathbf{y}_{4}=\left[\begin{array}{ll}
\mathbf{I}_{2} & \mathbf{0}_{2}
\end{array}\right] \mathbf{x}_{4}=\mathbf{C}_{4}^{T} \mathbf{x}_{4} .
\end{aligned}
$$

where $\mathbf{x}^{4} \in \mathrm{R}^{4}$ is a vector of system states, $\mathbf{y}_{4}(\mathbf{t}) \stackrel{\Delta}{=}[\alpha(t) h(t)]^{\mathrm{T}}$ denotes the output vector, $\mathbf{u}(\mathbf{t}) \in \mathrm{R}^{2}$ denotes the control input vector, $\overline{\mathbf{W}}_{4}\left(\mathbf{y}_{4}, \boldsymbol{\varphi}_{4}\right) \in \mathrm{R}^{4}$ is an auxiliary vector that captures the system nonlinearities, $\mathbf{G}_{4, \mu} \in \mathrm{R}^{2 \times 2}$ is a constant non-singular (but sign-indefinite), non-symmetric, gain matrix. Note that the notation $0 \mathrm{j}$ and $\mathrm{Ij}$ denote, respectively, the zero and identity matrices of dimension j. $\overline{\mathbf{W}}_{4}\left(\mathbf{y}_{4}, \boldsymbol{\varphi}_{4}\right)$ and $\mathrm{g}_{4, \mathrm{jk}}$ have been explicitly defined in Reddy et al. (2007). In Eq. (53), $\overline{\mathbf{W}}_{4}\left(\mathbf{y}_{4}, \boldsymbol{\varphi}_{4}\right)$ can be linearly parameterized as follows

$$
\begin{aligned}
\overline{\mathbf{W}}_{4}\left(\mathbf{y}_{4}, \varphi_{4}\right) & =\mathbf{W}_{4}\left(\mathbf{y}_{4}\right) \varphi_{4}=\sum_{j=1}^{p_{4}} \varphi_{4, j} \mathbf{W}_{4, j}\left(\mathbf{y}_{4}\right) \\
& =\sum_{j=1}^{p_{4}} \varphi_{4, j}\left[\begin{array}{llll}
W_{4, j, 1}\left(\mathbf{y}_{4}\right) & W_{4, j, 2}\left(\mathbf{y}_{4}\right) & W_{4, j, 3}\left(\mathbf{y}_{4}\right) & W_{4, j, 4}\left(\mathbf{y}_{4}\right)
\end{array}\right]^{T}
\end{aligned}
$$

where $\boldsymbol{\varphi}_{4} \in \mathrm{R}^{p_{4}}$ is a vector of constant unknowns while $\varphi_{4, j}$ is the jth element in $\boldsymbol{\varphi}_{4}, \mathbf{W}_{4, j}\left(\mathbf{y}_{4}\right) \in \mathrm{R}^{4} x^{p_{4}}$ is the $j$ th column of measurable nonlinear regression matrix $\mathbf{W}_{4}\left(\mathrm{y}_{4}\right)$. Assuming that both the leading principal minors $\mathrm{g}_{4,11}$ and $\Delta=\operatorname{det}\left(\mathbf{G}_{4, \mu}\right)$ of the matrix $\mathbf{G}_{4, \mu}$ are non-zero, a matrix decomposition introduced in Morse (1993) can be utilized to obtain the following result

$$
\mathbf{G}_{4, \mu}=\mathbf{S}_{4} \mathbf{D}_{4} \overline{\mathbf{U}}_{4}
$$

where $\mathbf{S}_{4}$ is a symmetric, positive-definite matrix, $\mathbf{D}_{4}$ is a diagonal matrix with its non-zero elements belonging to the set $\{+1,-1\}$, and $\overline{\mathbf{U}}_{4}$ is a unity upper triangular matrix. For purposes of control design, it's assumed that the signs of the leading principal minors of the high-frequency gain matrix $\mathbf{G}_{4, \mu}$ are known, i.e., the diagonal matrix $\mathbf{D}_{4}$ is assumed to be known. The tracking error $\mathbf{e}_{4}(\mathbf{t}) \in \mathrm{R}^{2}$, state estimation error $\boldsymbol{\varepsilon}_{4}(\mathbf{t})=\left[\begin{array}{ll}\boldsymbol{\varepsilon}_{4, \mu}^{T} & \boldsymbol{\varepsilon}_{4, b}^{T}\end{array}\right]^{T} \in \mathrm{R}^{4}$, and parameter estimation error signals $\tilde{\psi}_{4.1}(t) \in \mathrm{R}^{p_{4,1}}, \tilde{\psi}_{4.2}(t) \in \mathrm{R}^{p_{4,2}}$ can be defined as follows

$$
\begin{array}{lll}
\mathbf{e}_{4} \equiv \mathbf{y}_{4}-\mathbf{y}_{4, d}, & \boldsymbol{\varepsilon}_{4} \equiv \mathbf{x}_{4}-\hat{\mathbf{x}}_{4}, \\
\tilde{\psi}_{4,1} \equiv \psi_{4,1}-\hat{\psi}_{4,1}, & \tilde{\psi}_{4,2} \equiv \psi_{4,2}-\hat{\psi}_{4,2}
\end{array}
$$

where $\mathbf{y}_{4, d}(t) \in \mathrm{R}^{2}$ denotes a sufficiently smooth, bounded desired output vector. $\hat{\mathbf{x}}_{4}(\mathbf{t})=\left[\begin{array}{ll}\hat{\mathbf{x}}_{4, \mu} & \hat{\mathbf{x}}_{4, b}^{T}\end{array}\right]^{T} \in \mathrm{R}^{4}$ is a state estimation vector that will be designed later. $\tilde{\boldsymbol{\psi}}_{4.1}(t) \in \mathrm{R}^{p_{4,1}}$ and $\tilde{\boldsymbol{\psi}}_{4.2}(t) \in \mathrm{R}^{p_{4,2}}$ are unknown constant vectors, while $\tilde{\boldsymbol{\psi}}_{4.1}(t) \in \mathrm{R}^{p_{4,1}}$ and $\tilde{\psi}_{4.2}(t) \in \mathrm{R}^{p_{4,2}}$ are their corresponding estimates that will be given subsequently. In Xing and Singh (2000), the entire state of the system is observable by only using the measurement of the pitch angle variable. However, in order to rapidly regulate the pitching and plunging displacements in an adaptive framework, both the pitching and plunging variables are assumed to be measurable in this problem. Following the approach in Krstic et al. (1995), the filter $\zeta_{4,0}=\left[\zeta_{4,0,1} \zeta_{4,0,2} \zeta_{4,0,3}\right.$ $\left.\zeta_{4,0,4}\right]^{T} \in \mathrm{R}^{4}$ can be designed as follows

$$
\begin{aligned}
& \dot{\zeta}_{4,0}=\mathbf{A}_{4} \zeta_{4,0}+\mathbf{K}_{4} \mathbf{C}_{4}^{T}\left(\mathbf{x}_{4}-\zeta_{4,0}\right), \\
& \dot{\zeta}_{4, j}=\mathbf{A}_{4,0} \zeta_{4, j}+\mathbf{W}_{4, j}\left(\mathbf{y}_{4}\right), \forall j=1 \ldots p_{4}, \\
& \dot{\boldsymbol{v}}_{4}=\left[\begin{array}{l}
\dot{\mathbf{v}}_{4, u} \\
\dot{\mathbf{v}}_{4, b}
\end{array}\right]=\mathbf{A}_{4,0} \mathbf{v}_{4}+\left[\begin{array}{l}
\mathbf{0}_{4,2} \\
\mathbf{I}_{4,2}
\end{array}\right] \mathbf{u}
\end{aligned}
$$

where $\mathbf{K}_{4}=\left[\begin{array}{ll}k_{01} \mathbf{I}_{2} & k_{02} \mathbf{I}_{2}\end{array}\right]^{T} \in \mathrm{R}^{4 \times 2}$ denotes a constant gain matrix where the scalars $k_{o 1}$ and $k_{o 2}$ are chosen such that $\mathbf{A}_{4.0}=\mathbf{A}_{4}-\mathbf{K}_{4} \mathbf{C}_{4}^{T}$ is Hurwitz. $\boldsymbol{v}_{4}=\left[\begin{array}{ll}v_{4, u}^{T} & v_{4, b}^{T}\end{array}\right] \in \mathrm{R}^{4}$. Also note that it is straightforward to guarantee the boundedness of the filter variables $\zeta_{4,0}(t), \zeta_{4, j}(t)$, and $\boldsymbol{v}_{4}(t)$ provided that $\mathbf{y}_{4}(t)$ and $\mathbf{u}(t)$ remain bounded. Given Eq. (57), an immeasurable state estimate $\hat{\mathbf{x}}_{4}(\mathbf{t})$ can be obtained as follows

$$
\hat{\mathbf{x}}_{4}=\zeta_{4,0}+\sum_{j=1}^{p_{4}} \varphi_{4, j} \zeta_{4, j}+\left[\begin{array}{cc}
\mathbf{G}_{4, \mu} & \mathbf{0}_{2} \\
\mathbf{0}_{2} & \mathbf{G}_{4, \mu}
\end{array}\right] \mathbf{U}_{4}
$$

By taking the derivative of state estimation error and substituting for the dynamics of Eqs. (53) and (57), the following exponentially stable estimation error system is obtained 


$$
\dot{\varepsilon}_{4}=\mathbf{A}_{4} \varepsilon_{4}
$$

By using the backstepping technique, the control input signal $\mathbf{u}(t)$ can be designed as

$$
\mathbf{u}=-\mathbf{D}_{4} \mathbf{Y}_{4,2} \hat{\Psi}_{4,2}
$$

where the unknown parameters in $\hat{\psi}_{4.2}(t)$ can be dynamically estimated as follows

$$
\dot{\hat{\psi}}_{4,2}=\Gamma_{4,2} \mathbf{Y}_{4,2}^{T} \tilde{\mathbf{v}}_{4, b}
$$

where $\mathbf{Y}_{4,2}(\cdot) \in \mathrm{R}^{2 \times p_{4,2}}$ is a measurable regression vector and $\mathbf{Y}_{4,2}(\cdot) \in \psi_{4,2} \in \mathrm{R}^{2}$ can be explicitly defined as follows

$$
\mathbf{Y}_{4,2} \boldsymbol{\psi}_{4,2}=\mathbf{k}_{4,2} \tilde{\mathbf{v}}_{4, b}-\overline{\mathbf{Y}}_{4,2}(.) \bar{\psi}_{4,2}-k_{o 2} \mathbf{D} \mathbf{v}_{4, u}+d_{4,2}\left(\frac{\partial \overline{\mathbf{v}}_{4, b d}}{\partial \mathbf{y}_{4}}\right)^{T}\left(\frac{\partial \overline{\mathrm{v}}_{4, b d}}{\partial \mathbf{y}_{4}}\right) \tilde{\mathrm{u}}_{4, b}+\mathbf{e}_{4}
$$

where $\mathbf{k}_{4,2} \in \mathrm{R}^{2 \times 2}$ is a control gain matrix while $d_{4,2}$ is a positive scalar gain. $\overline{\mathbf{Y}}_{4,2}(\cdot) \overline{\boldsymbol{\psi}}_{4,2}$ have been explicitly defined in

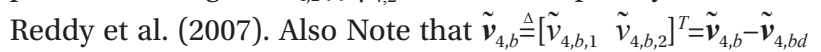
while $\tilde{\boldsymbol{v}}_{4, b}=\left[\begin{array}{ll}\tilde{v}_{4, b, 1} & \tilde{v}_{4, b, 2}\end{array}\right]^{T \Delta}=\mathbf{D}_{4} \boldsymbol{v}_{4, b} \in \mathrm{R}^{2}$ is an auxiliary measurable vector. $\tilde{\boldsymbol{v}}_{4, b d}=\left[\begin{array}{ll}\tilde{v}_{4, b d, 1} & \tilde{v}_{4, b d, 2}\end{array}\right]^{T} \in \mathrm{R}^{2}$ is a virtual control input that can be designed as follows

$$
\overline{\mathbf{v}}_{4, b d}=-\mathbf{Y}_{4,1}\left(\mathbf{y}_{4, d}, \dot{\mathbf{y}}_{4, d}, \mathbf{y}_{4}, \zeta_{4,0}, \zeta_{4, j},\left(\overline{\mathbf{U}}_{4}-\mathbf{I}_{2}\right) \overline{\mathbf{v}}_{4, b}\right) \hat{\psi}_{4,1}
$$

where $\hat{\boldsymbol{\psi}}_{4,1}(t)$ is dynamically generated as follows

$$
\dot{\hat{\psi}}_{4,1}=\Gamma_{4,1} \mathbf{Y}_{4,1}^{T} \mathbf{e}_{4} \text {. }
$$

Note that $\mathbf{Y}_{4,1}\left(\mathbf{y}_{4, d}, \dot{\mathbf{y}}_{4 d}, \mathbf{y}_{4}, \zeta_{4,0}, \zeta_{4, j},\left[\tilde{v}_{4, b, 2} 0\right]^{T}\right) \in \mathrm{R}^{2 \times p_{4,1}}$ is a measurable regression vector, $\overline{\boldsymbol{\psi}}_{4,1} \in \mathrm{R}^{p_{4,1}}$ is an unknown parameter vector and $\mathbf{Y}_{4,1}(\cdot) \boldsymbol{\psi}_{4,1}$ is explicitly defined as follows

$$
\begin{aligned}
\mathbf{Y}_{4,1} \Psi_{4,1}= & \mathbf{S}_{4}^{-1}\left[\left[\begin{array}{c}
\zeta_{4,0,3} \\
\zeta_{4,0,4}
\end{array}\right]+\sum_{j=1}^{p_{4}} \varphi_{4, j}\left(\left[\begin{array}{l}
\zeta_{4, j, 3} \\
\zeta_{4, j, 4}
\end{array}\right]+\left[\begin{array}{l}
\mathbf{W}_{4, j, 1}\left(\mathbf{y}_{4}\right) \\
\mathbf{W}_{4, j, 2}\left(\mathbf{y}_{4}\right)
\end{array}\right]\right)\right] \\
& +\left(\overline{\mathbf{U}}_{4}-\mathbf{I}_{2}\right) \overline{\mathbf{U}}_{4, b}-\mathbf{S}_{4}^{-1} \dot{\mathbf{y}}_{4, d}+\mathbf{k}_{4,1} \mathbf{e}_{4}+d_{4,1} \mathbf{e}_{4}
\end{aligned}
$$

where $\mathbf{k}_{4,1} \in \mathrm{R}^{2 \times 2}$ is a control gain matrix and $d_{4,1}$ is a positive scalar. From the Lyapunov stability analysis given in Reddy et al. (2007), one can easily show that $\lim \mathbf{e}_{4}(t), \tilde{\boldsymbol{v}}_{4, b}(t), \boldsymbol{\varepsilon}_{4}(t)=0$. Thus, the system is globally asymptotically stable and all signals in the plant, estimator, and controller stay bounded in closed-loop operation.

\subsection{Model-free output control in aeroelastic system subject to external disturbance}

Motivated by Chen et al. (2008), the governing Eqs. (1) and (3) can be transformed into the following input-output representation to facilitate the model-free output feedback design

$$
\ddot{\mathbf{x}}_{5}=\mathbf{h}_{5}\left(\mathbf{x}_{5}, \dot{\mathbf{x}}_{5}\right)+\mathbf{w}_{5, h d}+\mathbf{G}_{5} \mathbf{u}
$$

where $\mathbf{x}_{5} \stackrel{\Delta}{=}[h, \alpha]^{T} \in \mathrm{R}^{2}$ is the system output vector, $\mathbf{u}=\left[u_{1}\right.$, $\left.u_{2}\right]^{T} \triangleq[\beta, \gamma]^{T} \in \mathrm{R}^{2}$ denotes the control input vector, $\mathbf{h}_{5}\left(\mathbf{x}_{5}, \dot{\mathbf{x}}_{5}\right)$ represents uncertain nonlinearities due to the existence of $k_{\alpha}(\alpha) . \mathbf{w}_{5, h d}$ is the bounded unknown external disturbance terms. $\mathbf{G}_{5} \in \mathrm{R}^{2 \times 2}$ is a constant non-singular gain matrix for which the constant matrix entries $g_{5, i j}$ are explicitly defined in Wang et al. (2010). Based on the matrix decomposition introduced in Morse (1993) and the facts that both the leading principal minors $g_{5,11}$ and $\Delta_{5}$ are non-zero, $\mathbf{G}_{5}$ can be decomposed as $\mathbf{G}_{5}=\mathbf{S}_{5} \mathbf{D}_{5} \mathbf{U}_{5}$ where $\mathbf{S}_{5}$ is a symmetric and positive-definite matrix, $\mathbf{D}_{5}$ is a diagonal matrix with diagonal entries +1 or $-1, \mathbf{U}_{5}$ is an unknown unity upper triangular matrix. For purposes of control design, it's assumed that the signs of the leading principal minors of the high-frequency gain matrix $\mathbf{G}_{5}$ are known. After applying the matrix decomposition property and multiplying both sides of Eq. (66) with $\mathbf{T}_{5}=\mathbf{S}_{5}^{-1} \in \mathrm{R}^{2 \times 2}$, Eq. (66) can be rewritten as

$$
\mathbf{T}_{5} \ddot{\mathbf{x}}_{5}=\mathbf{f}_{5}\left(\mathbf{x}_{5}, \dot{\mathbf{x}}_{5}\right)+\mathbf{w}_{5, d}+\mathbf{D}_{5} \mathbf{U}_{5} \mathbf{u}
$$

where $\mathbf{T}_{5}$ is a symmetric, positive definite matrix, $\mathbf{f}_{5}(\cdot) \stackrel{\Delta}{=} \mathbf{S}_{5}^{-1} \mathbf{h}_{5}(\cdot) \in \mathrm{R}^{2}$ contains unmodeled nonlinearities, while $\mathbf{w}_{5, d} \stackrel{\Delta}{=} \mathbf{S}_{5}^{-1} \mathbf{w}_{5, h d} \in \mathrm{R}^{2}$ represents bounded unknown external disturbance terms.

The tracking error $\mathbf{e}_{5,1}(t) \in \mathrm{R}^{2}$ for the aeroelastic system can be defined as $\mathbf{e}_{5,1} \stackrel{\Delta}{=} \mathbf{x}_{5, d}-\mathbf{x}_{5}$ where $\mathbf{x}_{5}$ has been previously defined in Eq. (66). Here, $\mathbf{x}_{5, d} \in \mathrm{R}^{2}$ is the desired output vector. To simplify the subsequent control design, the following auxiliary error signals $\mathbf{e}_{5,2}(t) \in \mathrm{R}^{2}$ and filtered tracking error $\mathbf{r}_{5}(t) \in \mathrm{R}^{2}$ are introduced as

$$
\mathbf{e}_{5,2}=\dot{\mathbf{e}}_{5,1}+\mathbf{e}_{5,1}, \quad \mathbf{r}_{5}=\mathbf{e}_{5,2}+\mathbf{e}_{5,1} .
$$

Assuming that both the output variable $\mathbf{x}_{5}$ and their first order time derivatives $\mathbf{x}_{5}$ can be measured directly, the following full-state feedback control law is proposed

$$
\mathbf{u}=\mathbf{D}_{5}^{-1}\left[\mathbf{K}_{5} \mathbf{r}_{5}+\hat{\mathbf{N}}_{5}-\mathbf{v}_{5}\right]
$$

where $\mathbf{K}_{5}=\mathbf{K}_{5, v}+\mathbf{K}_{5, d}+\mathbf{K}_{5, \lambda}$ with $\mathbf{K}_{5, v}=k_{5, v} \mathbf{I}_{2 \times 2}, \quad \mathbf{K}_{5, d}=k_{5, d} \mathbf{I}_{2 \times 2}$, $\mathbf{K}_{5, \lambda}=\operatorname{diag}\left\{k_{5, \Lambda}, 0\right\}$ while $\hat{\mathbf{N}}_{5}$ and $\mathbf{v}_{5}$ represent the feedforward compensator and robustifying term, respectively, to be designed later. $k_{5, v}, k_{5, d}$, and $k_{5, \Lambda}$ are the diagonal elements for matrices $\mathbf{K}_{5, v}, \mathbf{K}_{5, d}$, and $\mathbf{K}_{5, \lambda}$. Here, the nonlinear target function $\mathbf{N}_{5} \in \mathrm{R}^{2}$ without external disturbance can be given as follows

$$
\mathbf{N}_{5}\left(\overline{\mathbf{x}}_{\mathbf{t}}\right)=\mathbf{T}\left(\ddot{\mathbf{x}}_{5, d}+2 \dot{\mathbf{e}}_{5,1}\right)-\mathbf{f}\left(\mathbf{x}_{5}, \dot{\mathbf{x}}_{5}\right)+\mathbf{K}_{5, d} \mathbf{e}_{5,2}-\left[\Phi_{5}, 0\right]^{T}
$$


where the input vector $\overline{\mathbf{x}}_{t} \in \mathbf{R}^{11}$ for the nonlinear target function can be defined as follows

$$
\overline{\mathbf{x}}_{t}=\left[\mathbf{x}^{T}, \dot{\mathbf{x}}^{\mathbf{T}}, \mathbf{x}_{d}^{T}, \dot{\mathbf{x}}_{d}^{T}, \ddot{\mathbf{x}}_{d}^{T},\|\hat{\mathbf{Z}}\|_{F}\right]^{T}
$$

and $\Phi_{5}=d_{5,2}^{-1} \bar{U}_{5,12}\left(\hat{N}_{5,2}-v_{5,2}\right)$. Note that $\mathbf{Z}$ will be designed subsequently. Here $d_{5,2}$ is the second diagonal element for the diagonal matrix $\mathbf{D}_{5}$. $\bar{U}_{5, i j}$ denotes the ijth element of the matrix $\overline{\mathbf{U}}_{5}=\mathbf{D}_{5} \mathbf{U}_{5}-\mathbf{D}_{5} \cdot \hat{N}_{5,1}$ and $v_{5, i}$ represent the ith element for vectors $\hat{\mathbf{N}}_{5}$ and $\mathbf{v}_{5}$, respectively.

In this section, the adaptive control designs cannot be applied since the model of the wing section and external disturbance are unknown. Thus, a neural network feedforward compensator $\hat{\mathbf{N}}_{5}$ along with robustifying term $\mathbf{v}_{5}$ are proposed to compensate for this target function $\mathbf{N}_{5}$ and the disturbance signal $\mathbf{w}_{5, \mathrm{~d}}$. The nonlinear target function $\mathbf{N}_{5}$ defined in Eq. (70) can be approximated as a three-layer neural network target function of the form (Lewis et al., 2004)

$$
\mathbf{N}_{5}\left(\overline{\mathbf{x}}_{5}\right)=\mathbf{W}_{n n}^{T} \sigma\left(\mathbf{V}_{n n}^{T} \overline{\mathbf{x}}_{5}\right)+\varepsilon\left(\overline{\mathbf{x}}_{5}\right)
$$

where $\overline{\mathbf{x}}_{5}=\left[1, \overline{\mathbf{x}}_{t}^{T}\right]^{T} \in \mathrm{R}^{p_{s, 1}+1}$ denotes the augmented input vector while $\boldsymbol{\sigma}(\cdot)$ is the activation function in the form of sigmoid function. $\mathbf{V}_{n n} \in \mathrm{R}^{\left(p_{s, 1}+1\right) \times p_{s, 2}}$ is the ideal first layer interconnection weights matrix while $\mathbf{W}_{n n} \in \mathrm{R}^{\left(p_{s, 2}+1\right) \times p_{5,3}}$ denotes the ideal second layer interconnection weights matrix. Here $p_{5,1}+1, p_{5,2}+1$, and $p_{5,3}$ are the number of node in the input layer, hidden layer, and output layer, respectively. It's also assumed that the ideal weights matrix $\mathbf{W}_{n n}$ and $\mathbf{V}_{n n}$ are constant and bounded such that $\left\|\mathbf{W}_{n n}\right\|_{F} \leq W_{n n B}$ and $\left\|\mathbf{V}_{n n}\right\|_{F} \leq V_{n n B}$, where $W_{n n B}$ and $V_{n n B}$ are positive constants and $\|\cdot\|_{F}$ denotes the Frobenius norm. $\boldsymbol{\varepsilon}\left(\overline{\mathbf{x}}_{5}\right)$ is the functional reconstruction error vector and assumed to be bounded in a compact set by $\left\|\boldsymbol{\varepsilon}\left(\overline{\mathbf{x}}_{5}\right)\right\|<\boldsymbol{\varepsilon}_{N}$ where $\boldsymbol{\varepsilon}_{N}$ is an unknown positive constant related to the number of hidden nodes $p_{5,2}+1$. Based on Eq. (72) and above definitions, the typical three-level neural networks compensator for target function $\mathbf{N}_{5}\left(\overline{\mathbf{x}}_{5}\right)$ is given in the following form

$$
\hat{\mathbf{N}}_{5}\left(\overline{\mathbf{x}}_{5}\right)=\hat{\mathbf{W}}_{n n}^{T} \sigma\left(\hat{\mathbf{V}}_{n n}^{T} \overline{\mathbf{x}}_{5}\right)
$$

where $\hat{\mathbf{W}}_{n n}$ and $\hat{\mathbf{V}}_{n n}$ are estimated weights. Motivated by Lewis et al. (2004), the estimated weights can be updated or learned through on-line weight tuning algorithms of the form

$$
\begin{aligned}
& \dot{\hat{\mathbf{W}}}_{n n}=\left(\mathbf{F}_{n n} \hat{\sigma}-\mathbf{F}_{n n} \hat{\sigma}^{\prime} \hat{\mathbf{V}}_{n n}^{T} \overline{\mathbf{x}}_{5}\right) \mathbf{r}_{5}^{T}-\kappa \mathbf{F}_{n n}\left\|\mathbf{r}_{5}\right\| \hat{\mathbf{W}}_{n n}, \\
& \dot{\hat{\mathbf{V}}}_{n n}=\mathbf{G}_{n n} \overline{\mathbf{x}}_{5}\left(\hat{\sigma}^{T} \hat{\mathbf{W}}_{n n} \mathbf{r}_{5}^{T}\right)^{T}-\kappa \mathbf{G}_{n n}\left\|\mathbf{r}_{5}\right\| \hat{\mathbf{V}}_{n n}
\end{aligned}
$$

where $\mathbf{F}_{n n} \in \mathrm{R}^{\left(p_{2}+1\right) \times\left(p_{2}+1\right)}$ and $\mathbf{G}_{n n} \in \mathrm{R}^{\left(p_{1}+1\right) \times\left(p_{1}+1\right)}$ are positive- definite, diagonal matrices, $k>0$ is a scalar design parameter, while $\hat{\boldsymbol{\sigma}}=\boldsymbol{\sigma}\left(\hat{\mathbf{V}}_{n n}^{T} \overline{\mathbf{x}}_{5}\right)$. The composite idea weight matrix $\mathbf{Z}_{n n}$, estimated weight matrix $\hat{\mathbf{Z}}_{n n}$, and the neural network weight mismatch matrix $\tilde{\mathbf{Z}}_{n n}$ are defined as follows

$$
\mathbf{Z}_{\mathrm{nn}} \equiv\left[\begin{array}{cc}
\mathbf{W}_{n n} & \mathbf{0} \\
\mathbf{0} & \mathbf{V}_{n n}
\end{array}\right] ; \quad \hat{\mathbf{Z}}_{\mathrm{nn}} \equiv\left[\begin{array}{cc}
\hat{\mathbf{W}}_{n n} & \mathbf{0} \\
\mathbf{0} & \hat{\mathbf{V}}_{n n}
\end{array}\right] ; \quad \tilde{\mathbf{Z}}_{n n} \equiv\left[\begin{array}{cc}
\tilde{\mathbf{W}}_{n n} & \mathbf{0} \\
\mathbf{0} & \tilde{\mathbf{V}}_{n n}
\end{array}\right]
$$

Note that $\tilde{\mathbf{Z}}_{n n}=\mathbf{Z}_{n n}-\hat{\mathbf{Z}}_{n n}$. According to the boundedness property for $\left\|\mathbf{W}_{n n}\right\|_{F}$ and $\left\|\mathbf{V}_{n n}\right\|_{F}$, it will be assumed that there exists a constant $Z_{n n B}$ such that $Z_{n n B}>\left\|\mathbf{Z}_{n n}\right\|_{F}$. Based on the definition on $Z_{n n B}$, the robustifying term $\mathbf{v}_{5} \in \mathrm{R}^{2}$ in Eq. (69) can be defined as

$$
\mathbf{v}_{5}=-K_{z}\left(\left\|\hat{\mathbf{Z}}_{n n}\right\|_{F}+Z_{n n B}\right) \mathbf{r}_{5}
$$

where $\mathrm{Kz}$ is a positive constant. Finally, it is noted that the unknown external disturbance wd and functional reconstruction error $\boldsymbol{\varepsilon}\left(\overline{\mathbf{x}}_{5}\right)$ are assumed to be bounded. Stability analysis given in Wang et al. (2010) shows that if the control gain matrix $\mathbf{K}_{5}$ defined in Eq. (69) is chosen to be appropriately large, the error signals $\left(\mathbf{r}_{5}\right.$ and $\tilde{\mathbf{Z}}_{n n}$ ) for the closed-loop system are uniformly ultimately bounded.

When the only measurements available are the pitching and plunging displacements, a high gain observer (HGO) is used to estimated the remaining states. Motivated by the result in Atassi and Khalil (1999), an estimate $\hat{\mathbf{z}}_{5}(t)=\left[\hat{\mathbf{e}}_{5,1}^{T}, \hat{\mathbf{e}}_{5,2}^{T}\right.$, $\left.\hat{\mathbf{r}}_{5}^{T}\right]^{T} \in \mathrm{R}^{6}$ for the auxiliary error signal $\mathbf{z}_{5}(t)$ can be obtained via the design of the following HGO

$$
\dot{\hat{\mathbf{e}}}_{5,1}=\hat{\mathbf{r}}_{5}-\mathbf{2} \hat{\mathbf{e}}_{5,1}+\frac{\alpha_{5,1}}{\bar{\varepsilon}}\left(\mathbf{e}_{5,1}-\hat{\mathbf{e}}_{5,1}\right) \text { and } \dot{\hat{\mathbf{r}}}_{5}=\frac{\alpha_{5,2}}{\bar{\varepsilon}^{2}}\left(\mathbf{e}_{5,1}-\hat{\mathbf{e}}_{5,1}\right)
$$

where $\alpha_{5, i} \forall i=1,2$ are gain constants and $\bar{\varepsilon}$ is a small positive constant. Note that $\hat{\mathbf{e}}_{5,2}=\hat{\mathbf{r}}_{5}-\hat{\mathbf{e}}_{5,1}$. In order to suppress the peaking phenomenon due to using HGO, the full-state control design of Eq. (69) can be modified into an output feedback saturated control as follows

$$
\mathbf{u}=\operatorname{sat}\left[\mathbf{D}_{5}^{-1}\left(\mathbf{K}_{5} \hat{\mathbf{r}}_{5}+\hat{\mathbf{N}}_{5}-\mathbf{v}_{5}\right)\right]
$$

where $\operatorname{sat}(\cdot)$ stands for standard saturation function and saturation is applied outside an appropriately defined compact set for the control input $\mathbf{u}$. Here $\hat{\mathbf{N}}_{5}$ and $\mathbf{v}_{5}$ have been defined in the same manner as in Eq. (69). For details of the stability analysis of the output feedback control design, the reader is referred to Atassi and Khalil (1999). Also note that according to the observer theory, there is a trade off between the speed of state reconstruction and the immunity to measurement noise (Ahrens and Khalil, 2009). As we know, the HGO could quickly reconstruct the unknown system states through applying a large enough observer gain. 
However, a large gain could also magnify the measurement noise (Vasiljevic and Khalil, 2008). A switch control strategy introduced in Ahrens and Khalil (2009) can be employed to address this problem.

\section{Numerical Simulations and Comparisons}

In this section, the simulation results for the 2-DOF aeroelastic wing section models with one and two control surfaces at subsonic/supersonic flight speed regimes were presented. The parameters for the wing section model with one control surface at subsonic speed were the same as the ones used in Ko et al. (1997) and Behal et al. (2006a), which are given in Table 1. For the supersonic wing section model in the nondimensional form, the parameters were chosen the same as Rao et al. (2006) and represented in the Table 2. The corresponding parameters for the subsonic wing section model with two control surfaces, which have been used in (Platanitis and Strganac, 2004; Reddy et al., 2007; Wang et al., 2010), are listed in Table 3. The desired trajectory $\mathbf{x}_{i, d}, \dot{\mathbf{x}}_{i, d}$, and $\ddot{\mathbf{x}}_{i, d}$, in the following sets of simulation were all simply selected as zero.

The first set of simulation, under the control designed in

Table 1. Wing section parameters used in Section 3.1

\begin{tabular}{clcl}
\hline Parameter & \multicolumn{1}{c}{ Value } & Parameter & \\
\hline$a$ & $=-0.4$ & $b$ & $=0.135 \mathrm{~m}$ \\
$I_{\alpha}$ & $=0.065 \mathrm{~kg} \cdot \mathrm{m}^{2}$ & $\rho_{\infty}$ & $=1.225 \mathrm{~kg} / \mathrm{m}^{3}$ \\
$m$ & $=12.387 \mathrm{~kg}$ & $x_{\alpha}$ & $=[0.07873-(\mathrm{b}+\mathrm{ab})] / \mathrm{b}$ \\
$C_{h}$ & $=27.43 \mathrm{~kg} / \mathrm{s}$ & $C_{\alpha}$ & $=0.036 \mathrm{~N} \cdot \mathrm{s}$ \\
$C_{l \alpha}$ & $=6.281 / \mathrm{rad}$ & $C_{m \alpha}$ & $=(0.5+\mathrm{a}) 1 / \mathrm{rad}$ \\
$C_{l \beta}$ & $=3.3581 / \mathrm{rad}$ & $C_{m \beta}$ & $=-0.6351 / \mathrm{rad}$ \\
$k_{h, i}$ & $=2844.4$ & $k_{\alpha, i}$ & $=[2.8,62.3,3709.7,24195.6,48756.9]$ \\
\hline
\end{tabular}

Table 2. Wing section parameters used in Section 3.2

\begin{tabular}{clcl}
\hline Parameter & Value & Parameter & Value \\
\hline$x_{\alpha}$ & $=0.25$ & $b$ & $=1.5 \mathrm{~m}$ \\
$C_{h} / m$ & $=0.01$ & $C_{\alpha} / I_{\alpha}$ & $=0.01$ \\
$x_{0}$ & $=0.5$ & $x_{1}$ & $=0.75$ \\
$\lambda$ & $=1$ & $\gamma$ & $=1.4$ \\
$r_{\alpha}$ & $=0.5$ & $U_{\infty}$ & $=565.2 \mathrm{~m} / \mathrm{s}$ \\
$\mu$ & $=50$ & $k_{\alpha, 3} / k_{\alpha, 1}$ & $=5$ \\
$\omega_{h}$ & $=376.8 \mathrm{rad} / \mathrm{s}$ & $\omega_{\alpha}$ & $=376.8 \mathrm{rad} / \mathrm{s}$
\end{tabular}

Table 3. Wing section parameters used in Section 3.3-3.5

\begin{tabular}{cccc}
\hline Parameter & Value & Parameter & Value \\
\hline$a$ & $=-0.6719$ & $b$ & $=0.1905 \mathrm{~m}$ \\
$s$ & $=0.5945 \mathrm{~m}$ & $\rho_{\infty}$ & $=1.225 \mathrm{~kg} / \mathrm{m}^{3}$ \\
$r_{c g}$ & $-\mathrm{b}(0.0998+\mathrm{a}) \mathrm{m}$ & $x_{\alpha}$ & $=\mathrm{rcg} / \mathrm{b}$ \\
$C_{h}$ & $=27.43 \mathrm{~kg} / \mathrm{s}$ & $C_{\alpha}$ & $=0.036 \mathrm{~N} \cdot \mathrm{s}$ \\
$k_{h, i}$ & $=2844.4$ & $m_{\text {wing }}$ & $=4.340 \mathrm{~kg}$ \\
$I_{c g w}$ & $=0.04342 \mathrm{~kg} \cdot \mathrm{m}^{2}$ & $I$ & $=0.04697 \mathrm{~kg} \cdot \mathrm{m}^{2}$ \\
$m_{w}$ & $=5.23 \mathrm{~kg}$ & $m_{T}$ & $=15.57 \mathrm{~kg}$ \\
$C_{l \alpha}$ & $=6.7571 / \mathrm{rad}$ & $C_{m \alpha}$ & $=01 / \mathrm{rad}$ \\
$C_{l \beta}$ & $=3.7741 / \mathrm{rad}$ & $C_{m \beta}$ & $=-0.67191 / \mathrm{rad}$ \\
$C_{l y}$ & $=-0.15661 / \mathrm{rad}$ & $C_{m \gamma}$ & $=-0.10051 / \mathrm{rad}$ \\
$k_{\alpha, i}$ & $=[12.77,53.47,1003]$ & $I_{\alpha}$ & $=I_{c a m}+I_{c g w}+m_{\text {wing }} r_{c g}^{2} \mathrm{~kg} \cdot \mathrm{m}^{2}$ \\
\hline
\end{tabular}




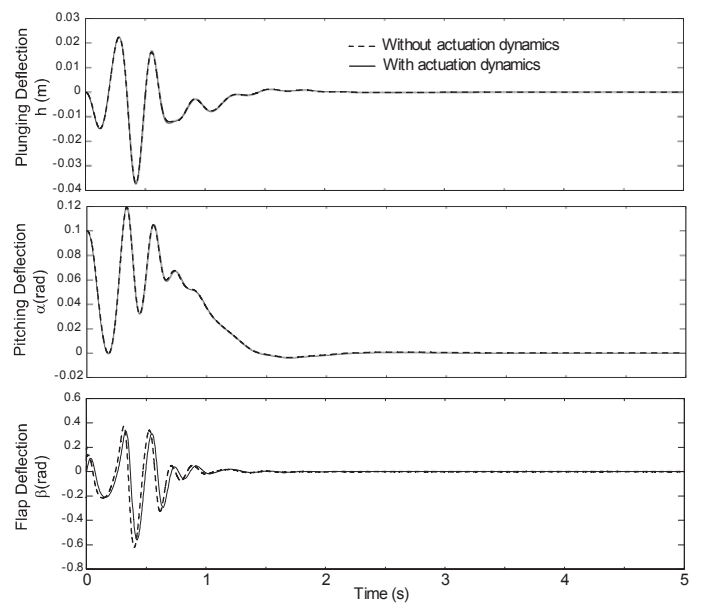

Fig. 2. Closed-loop aeroelastic responses with and without actuation dynamics $U_{\infty}=20 \mathrm{~m} / \mathrm{s}$.

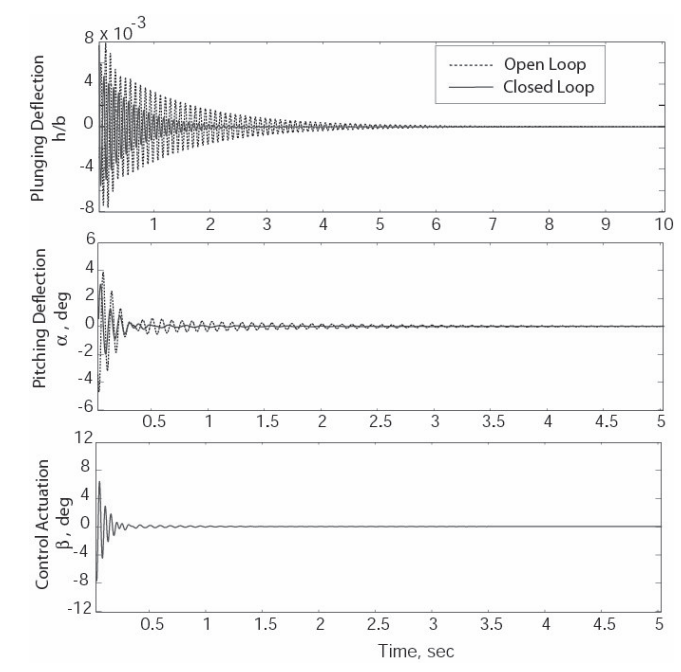

Fig. 3. Open-/closed-loop plunging, pitching and control deflections time-histories in subcritical flight speed regime, $\bar{M}=2<\bar{M}_{\text {futter }}=2.15$.

Section III-A, was run at freestream velocity $U_{\infty}=20 \mathrm{~m} / \mathrm{s}$ on the subsonic wing section model with only TECS available. The initial conditions for the system states were selected as $\alpha(0)$ $=0.1 \mathrm{rad}, \mathrm{h}(0)=0 \mathrm{~m}$, and state estimates values were simply set to zero. Figure 2 demonstrates the closed-loop plunging, pitching, and control surface deflection when the actuation was turned on at time zero. It's straightforward to see that in the presence of active control, the system states were stabilized to zero fairly rapidly. Also note that the proposed adaptive control strategy was able to quickly regulate the plunging and pitching displacements with a large parametric uncertainty. When the fast actuator dynamics was introduced, the difference in pitching time history was imperceptible from when there were no dynamics considered, since the poles of

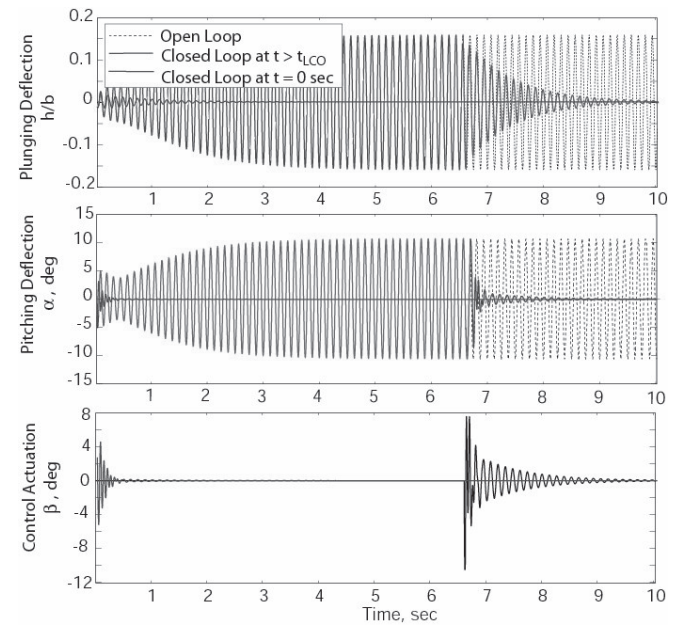

Fig. 4. Open-/closed-loop plunging, pitching and control deflections time-histories in supercritical flight speed regime, $\bar{M}=3$.

the actuation dynamics did not interact with the closed-loop poles of the pitching and plunging dynamics. However, one could expect that the inclusion of slow actuator dynamics would degrade transient performance.

When the trailing edge flap was actuated at time $t=0 \mathrm{~s}$, Figure 3 illustrates the open and closed loop plunging and pitching deflection associated with the control input signal design in Section 3.2 for the supersonic aeroelastic model given in Eqs. (1), (8), and (9). With the following initial conditions: $\xi=h / b=0.1 ; \alpha=0.0 \mathrm{rad}$, the first set of simulation was carried out in the subcritical flight speed regime, $\bar{M}=2<\bar{M}_{\text {flutter }}=2.15$. From Fig. 3 , oscillations in the pitching axis can be suppressed within $1 \mathrm{~s}$ while the vibrations in the plunging axis were lightly damped and can be suppressed only in about $2.5 \mathrm{~s}$. A second set of simulations is shown in Fig. 4 for a freestream velocity above the flutter speed, $\bar{M}=3>\bar{M}_{\text {flutter }}=2.15$ where two cases have been considered. In the first case, the control was turned on from $t=0 \mathrm{~s}$, while in the second case, the control was turned on at $t=6.5 \mathrm{~s} \mathrm{such}$ that an LCOs was experienced due to the non-linear pitch stiffness and the aerodynamic nonlinearities. It's clear to see that the control was very effective if it was turned on at $t=0$ $\mathrm{s}$ and the oscillations could be suppressed within $1 \mathrm{~s}$. When the control was turned on $t=6.5 \mathrm{~s}$, the proposed control was still able to rapidly stabilize the pitching displacement within $1.5 \mathrm{~s}$ and the plunging displacement within $4 \mathrm{~s}$. Also note that in above cases that the control deflection remained within the actuator capabilities. Simulation results clearly showed that the adaptive control strategy was able to quickly stabilize the pitching and plunging responses even in the presence of large uncertainty in the parameters.

The model described in Fig. 1 was also simulated using the 
(a)

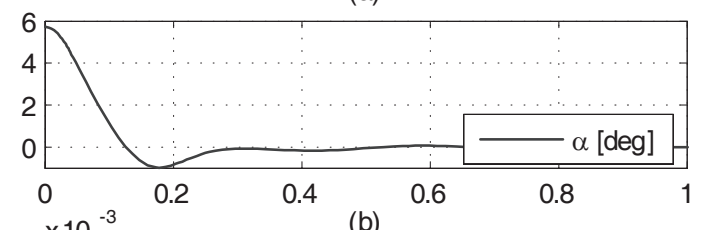

(b)

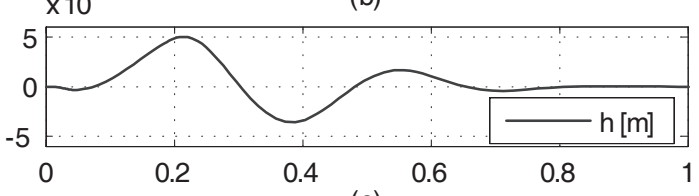

(c)

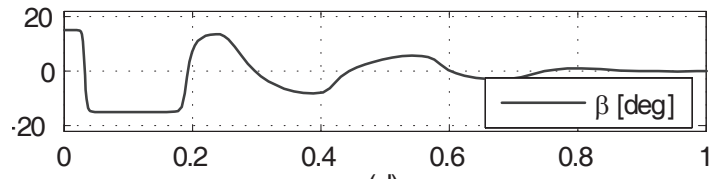

(d)

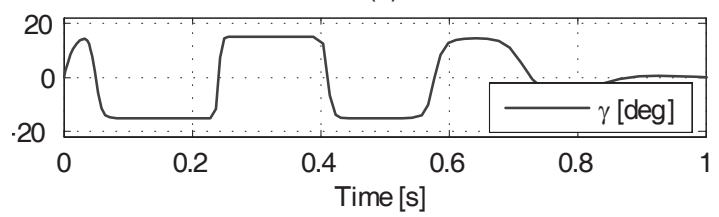

Fig. 5. Closed-loop pitching, plunging, and control surface deflections with both trailing edge control surface and leading edge control surface actuations for $\mathrm{U}_{\infty}=8 \mathrm{~m} / \mathrm{s}<\mathrm{U}_{\infty}=11.4 \mathrm{~m} / \mathrm{s}$.

(a)

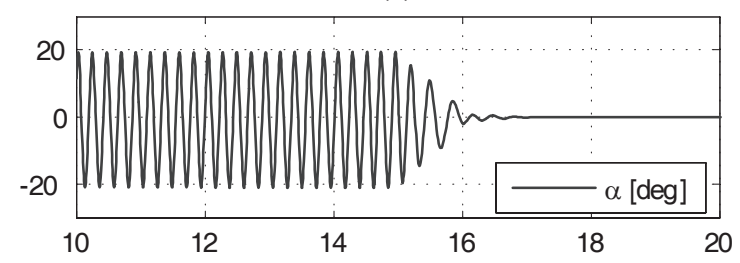

(b)

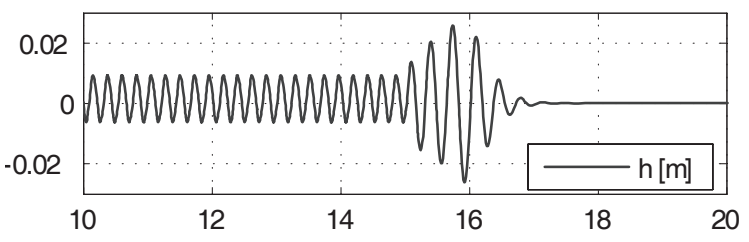

(c)

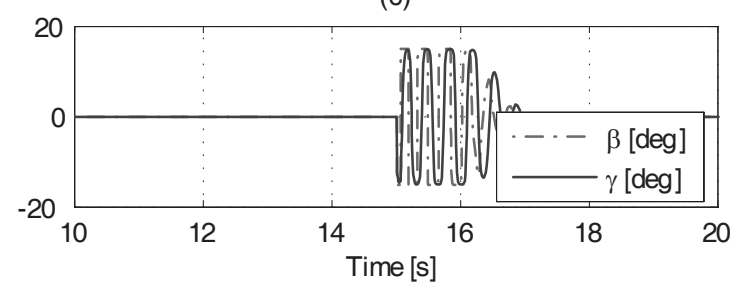

Fig. 6. Closed-loop pitching, plunging, and control surface deflections with both trailing edge control surface and leading edge control surface actuations for $U_{\infty}=13.15 \mathrm{~m} / \mathrm{s}>U_{\infty}=11.4 \mathrm{~m} / \mathrm{s}$. controller defined in Section 3.3. The model parameters have been chosen to be identical to those utilized in Platanitis and Strganac (2004), and both the TECS and LECS were constrained to vary between $\pm 15 \mathrm{deg}$. The initial conditions for the system states were selected as $\alpha(0)=0.1 \mathrm{rad}, h(0)=$ $0 \mathrm{~m}, \dot{\alpha}(0)=0 \mathrm{rad} / \mathrm{s}$, and $\dot{h}(0)=0 \mathrm{~m} / \mathrm{s}$, while the initial values for the parameter estimates were selected as $\hat{\theta}_{3, i}(0)=0, \forall i=1$, $\ldots, 17$ and $\hat{\theta}_{3, s}(0)=0.0049$. The first simulation was run with a freestream velocity of $U_{\infty}=8 \mathrm{~m} / \mathrm{s}<U_{\infty}=11.4 \mathrm{~m} / \mathrm{s}$. Given both the trailing and leading edge flaps were turned on at time $t=$ $0 \mathrm{~s}$, the vibrations in plunging and pitching were suppressed within $1 \mathrm{~s}$ in Fig. 5. A second set of simulations was carried out with similar initial conditions for freestream velocities at $U_{\infty}=13.15 \mathrm{~m} / \mathrm{s}$ above the flutter velocity. Initially, the system was allowed to evolve without control to produce LCOs until $t=15 \mathrm{~s}$. It can be seen, Fig. 6, that both the pitching and plunging LCOs were suppressed within $2 \mathrm{~s}$.

Figure 7 demonstrates the responses when both the LECS and TECS were actuated at $t=0 \mathrm{~s}$ under the control designed in Section 3.4 at $U_{\infty}=8 \mathrm{~m} / \mathrm{s}$. The initial conditions for pitch angle $\alpha(t)$ and plunge displacement $h(t)$ were chosen as $\alpha(0)$ $=0.1 \mathrm{rad}$ and $h(0)=0 \mathrm{~m}$ while all other variables $\dot{h}(t), \dot{\alpha}(t), \ddot{h}(t)$, $\ddot{\alpha}(t)$, state estimate variable, as well as unknown parameters $\hat{\psi}_{4.1}(t)$ and $\hat{\psi}_{4.2}(t)$ were initially set to zero. Both the leading edge $\beta(t)$ and trailing edge $\gamma(t)$ flaps were constrained to vary between \pm 15 deg. It can be seen that both the pitch angle and plunge displacements were settled down in less than $1 \mathrm{~s}$. The second simulation under the same control law was run at post-flutter velocity i.e., $U_{\infty}=13.15 \mathrm{~m} / \mathrm{s}$ with same initial conditions. In order to obtain the LCOs, the control was turn off until $t=15 \mathrm{~s}$. At $t=15 \mathrm{~s}$, both the trailing and leading edge actuators were switched on and the pitching LCOs was suppressed faster than plunging LCOs, whereas the plunging LCOs took around $2 \mathrm{~s}$ to settle in Fig. 8.

Finally, the simulation results for the algorithms introduced in Section III-E are presented. The initial conditions for pitch angle $\alpha(\mathrm{t})$ and plunge displacement $h(t)$ were chosen as $\alpha(0)$ $=0.1 \mathrm{rad}$ and $\mathrm{h}(0)=0 \mathrm{~m}$ while all other variables $\dot{h}(t), \dot{\alpha}(t)$, $\ddot{h}(t)$ and $\ddot{\alpha}(t)$ were initially set to zero. Since $\mathbf{x}_{d}, \dot{\mathbf{x}}_{d}$, and $\ddot{\mathbf{x}}_{d}$ were all bounded signals and chosen to be zero for all time, they can be removed from the input set $\overline{\mathbf{x}}_{t}$. Thus, the simplified input set can be given as $\overline{\mathbf{x}}_{t}=\left[\mathbf{x}^{T}, \dot{\mathbf{x}}^{T},\|\hat{\mathbf{Z}}\|_{F}\right]^{T} \in \mathrm{R}^{5} \cdot p_{1}, p_{2}$, and $p_{3}$ were given as $p_{1}=5, p_{2}=10$, and $p_{3}=2$. The number of hidden layer nodes was chosen through a trial and error method in order to obtain best performance. In the following sets of simulation, both the leading edge $\beta(t)$ and trailing edge $\gamma(t)$ flaps were constrained to vary between $\pm 15 \mathrm{deg}$. The initial weight $\hat{\mathbf{W}}$ is chosen to be zero while the initial weight for $\hat{\mathbf{V}}$ is randomly chosen between -1 and +1 . Under the proposed model-free control, three sets of simulations were carried 
(a)
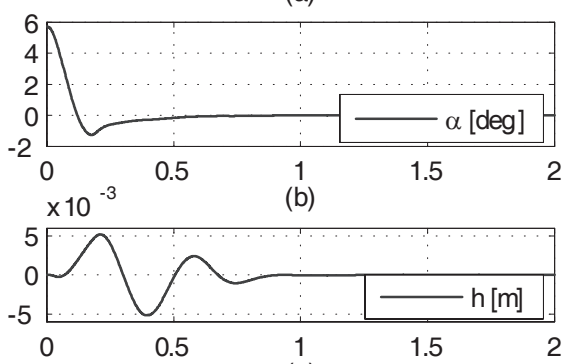

(c)

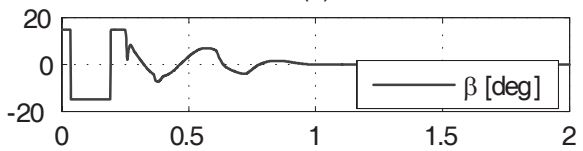

(d)

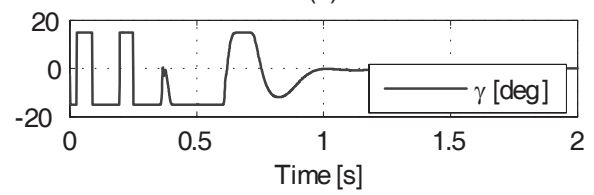

Fig. 7. Closed-loop pitching, plunging, and control surface deflections with both trailing edge control surface and leading edge control surface actuations for $U_{\infty}=8 \mathrm{~m} / \mathrm{s}<U_{F}=11.4 \mathrm{~m} / \mathrm{s}$.

(a)

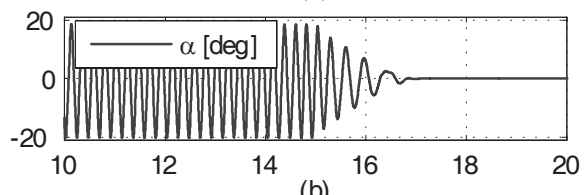

(b)

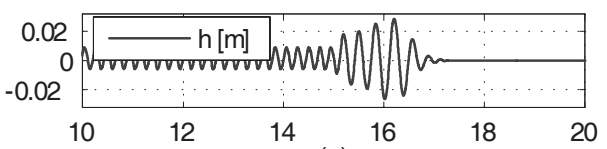

(c)
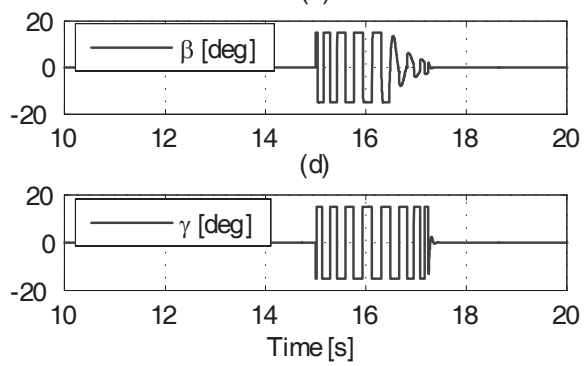

Fig. 8. Closed-loop pitching, plunging, and control surface deflections with both trailing edge control surface and leading edge control surface actuations for $U_{\infty}=13.15 \mathrm{~m} / \mathrm{s}>U_{F}=11.4 \mathrm{~m} / \mathrm{s}$.

out based on three types of external disturbances introduced in (Leishman, 1994). Under the triangular gust disturbance defined in Eq. (11), Fig. 9 illustrates that the proposed modelfree control drove the plunge and pitch displacements to zero in less than $3 \mathrm{~s}$ at pre-flutter speed. At post flutter speed $U_{\infty}=13.28 \mathrm{~m} / \mathrm{s}>U_{F}=11.4 \mathrm{~m} / \mathrm{s}$, the closed loop response of
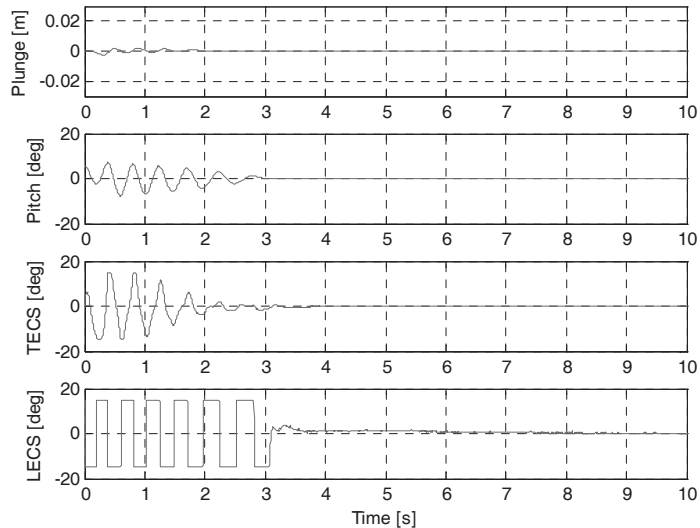

Fig. 9. Closed-loop pitching, plunging, and control surface deflections with both trailing edge control surface and leading edge control surface actuations for $U_{\infty}=8 \mathrm{~m} / \mathrm{s}<U_{F}=11.4 \mathrm{~m} / \mathrm{s}$ under triangular gust.
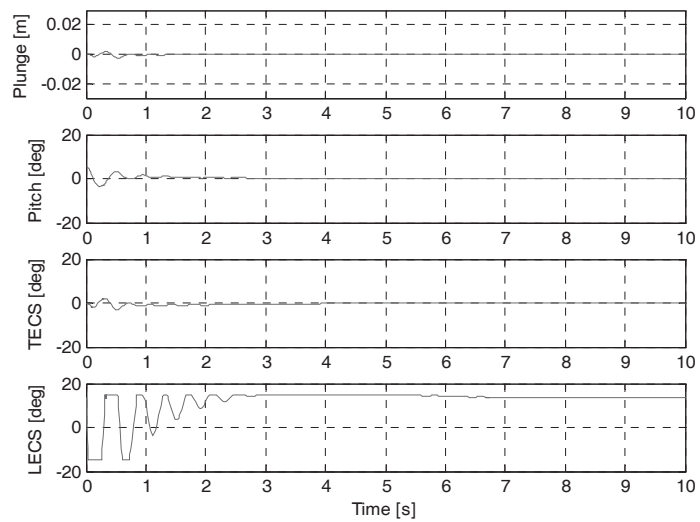

Fig. 10. Closed-loop pitching, plunging, and control surface deflections with both trailing edge control surface and leading edge control surface actuations for $U_{\infty}=13.28 \mathrm{~m} / \mathrm{s}>U_{F}=11.4$ $\mathrm{m} / \mathrm{s}$ under graded gust.
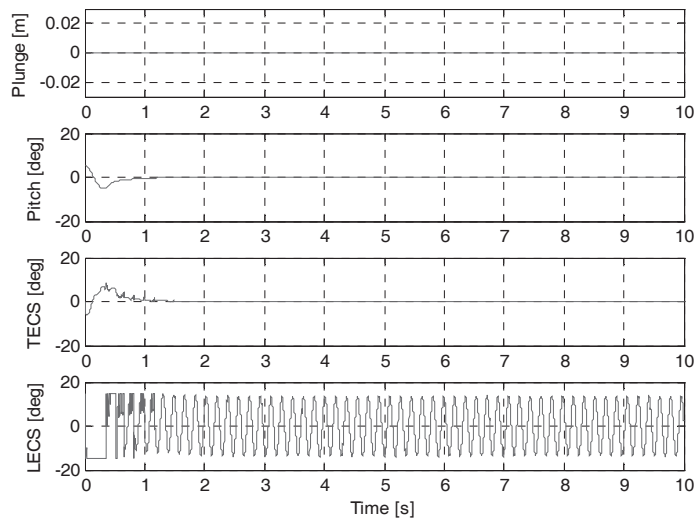

Fig. 11. Closed-loop pitching, plunging, and control surface deflections with both trailing edge control surface and leading edge control surface actuations for $U_{\infty}=13.28 \mathrm{~m} / \mathrm{s}>U_{F}=11.4$ $\mathrm{m} / \mathrm{s}$ under sinusoidal gust. 
the system under graded gust given in Eq. (12) are given in Fig. 10. One can easily see the convergence of the plunging and pitching displacements to the origin under the proposed method. Under a sinusoid-like gust represented in Eq. (13) at post flutter speed $U_{\infty}=13.28 \mathrm{~m} / \mathrm{s}>U_{F}=11.4 \mathrm{~m} / \mathrm{s}$, one can see that the proposed model-free controller successfully suppressed plunging and pitching displacements in less than 1.5 s from Fig. 11.

\section{Conclusions}

In this paper, several control strategies have been reviewed and represented with focus on adaptive control schemes. The state estimation methods, control design, and stability analysis results are presented in some detail, and are elaborated from the authors' contributions to the literature. All these control strategies are focused on the advanced single-input and multi-input multi-output adaptive feedback control strategies developed for lifting surfaces operating at subsonic and supersonic flight speeds. The corresponding concepts involving backstepping adaptive control design and neural networks are also presented in above paper From the theoretical and numerical results, the efficacy of the proposed methods toward suppressing aeroelastic vibration and LCOs in supersonic/subsonic flight speed is demonstrated.

This paper intends to contribute to the bulk of knowledge of active aeroelastic control of lifting surfaces. Advanced adaptive control system architectures sensing the changes in flight environment, automatically schedule the control gains and feedback to promote more optimal response characteristics to suppress flutter instabilities and limit cycle oscillations, and improved aerodynamic efficiency will be required to enhance maneuverability and operational effectiveness of revolutionary air vehicles.

\section{Acknowledgements}

P. Marzocca acknowledges the National Science Foundation (Grant No. CMMI-1031036) for Providing Partial funding for this research.

\section{References}

Ahrens, J. H. and Khalil, H. K. (2009). High-gain observers in the presence of measurement noise: A switched-gain approach. Automatica, 45, 936-943.

Atassi, A. N. and Khalil, H. K. (1999). A separation principle for the stabilization of a class of nonlinear systems. IEEE Transactions on Automatic Control, 44, 1672-1687.

Behal, A., Marzocca, P., Rao, V. M., and Gnann, A. (2006a). Nonlinear adaptive control of an aeroelastic two-dimensional lifting surface. Journal of Guidance, Control, and Dynamics, 29, 382-390.

Behal, A., Rao, V. M., Marzocca, P., and Kamaludeen, M. (2006b). Adaptive control for a nonlinear wing section with multiple flaps. Journal of Guidance, Control, and Dynamics, 29, 744-749.

Block, J. J. and Strganac, T. W. (1998). Applied active control for a nonlinear aeroelastic structure. Journal of Guidance, Control, and Dynamics, 21, 838-845.

Breitbach, E. (1978). Effects of Structural Non-Linearities on Aircraft Vibration and Flutter, AGARD Report No. 665. Neuily-sur-Seine, France: NATO Advisory Group for Aerospace Research and Development.

Chen, J., Behal, A., and Dawson, D. M. (2008). Robust feedback control for a class of uncertain MIMO nonlinear systems. IEEE Transactions on Automatic Control, 53, 591596.

Dowell, E. H. and Clark, R. (2004). A Modern Course in Aeroelasticity. 4th ed. Boston: Kluwer Academic Publishers.

Eastep, F. E. and Olsen, J. J. (1980). Transonic flutter analysis of a rectangular wing with conventional airfoil sections. AIAA Journal, 18, 1159-1164.

Edwards, J. W., Bennett, R. M., Whitlow Jr, W., and Seidel, D. A. (1983). Time-marching transonic flutter solutions including angle-of-attack effects. Journal of Aircraft, 20, 899906.

Fung, Y. C. (2002). An Introduction to the Theory of Aeroelasticity. Mineola: Dover Publications.

Gangsaas, D., Ly, U., and Norman, D. C. (1981). Practical gust load alleviation and flutter suppression control laws based on a lqg methodology. 19th AIAA Aerospace Sciences Meeting, St Louis, MO. AIAA-1981-0021.

Gujjula, S., Singh, S. N., and Yim, W. (2005). Adaptive and neural control of a wing section using leading- and trailingedge surfaces. Aerospace Science and Technology, 9, 161171.

Hariharan, N. and Leishman, J. G. (1996). Unsteady aerodynamics of a flapped airfoil in subsonic flow by indicial concepts. Journal of Aircraft, 33, 855-868.

Heeg, J. (1993). Analytical and experimental investigation of flutter suppression by piezoelectric actuation. NASA Technical Paper, 3241.

Horikawa, H. and Dowell, E. H. (1979). An elementary explanation of the flutter mechanism with active feedback controls. Journal of Aircraft, 16, 225-232.

Karpel, M. (1982). Design for active flutter suppression 
and gust alleviation using state-space aeroelastic modeling Journal of Aircraft, 19, 221-227.

Ko, J., Kurdila, A. J., and Strganac, T. W. (1997). Nonlinear control of a prototypical wing section with torsional nonlinearity. Journal of Guidance, Control, and Dynamics, 20, 1181-1189.

Ko, J., Strganac, T. W., and Kurdila, A. J. (1998). Stability and control of a structurally nonlinear aeroelastic system. Journal of Guidance, Control, and Dynamics, 21, 718-725.

Ko, J., Strganac, T. W., and Kurdila, A. J. (1999). Adaptive feedback linearization for the control of a typical wing section with structural nonlinearity. Nonlinear Dynamics, 18, 289-301.

Krstic, M., Kanellakopoulos, I., and Kokotovic, P. V. (1995). Nonlinear and Adaptive Control Design. New York: Wiley.

Lazarus, K. (1992). Multivariable High-Authority Control of Plate-Like Active Lifting Surfaces. PhD Thesis, Massachusetts Institue of Technology.

Lee, K. W. and Singh, S. N. (2007). Global robust control of an aeroelastic system using output feedback. Journal of Guidance, Control, and Dynamics, 30, 271-275.

Lee, K. W. and Singh, S. N. (2009). Immersion- and invariance-based adaptive control of a nonlinear aeroelastic system. Journal of Guidance, Control, and Dynamics, 32, 1100-1110.

Leishman, J. G. (1988). Validation of approximate indicial aerodynamic functions for two-dimensional subsonic flow. Journal of Aircraft, 25, 914-922.

Leishman, J. G. (1994). Unsteady lift of an airfoil with a trailing-edge flap based on indicial concepts. Journal of Aircraft, 31, 288-297.

Lewis, F. L., Abdallah, C. T., and Dawson, D. M. (2004). Robot Manipulator Control: Theory and Practice. 2nd ed. New York: Marcel Dekker.

Librescu, L. and Marzocca, P. (2005). Advances in the linear/nonlinear control of aeroelastic structural systems. Acta Mechanica, 178, 147-186.

Lin, C. Y. (1993). Strain Actuated Aeroelastic Control. MS Thesis, Massachusetts Institue of Technology.

Lyons, M. G., Vepa, R., McIntosh, S. C., and DeBra, D. B. (1973). Control law synthesis and sensor design for active flutter suppression. Proceedings of the AIAA Guidance and Control Conference, Key Biscayne, FL. AIAA Paper No 73832.

Marzocca, P., Librescu, L., and Chiocchia, G. (2001). Aeroelastic response of 2-D lifting surfaces to gust and arbitrary explosive loading signatures. International Journal of Impact Engineering, 25, 41-65.

Marzocca, P., Librescu, L., and Chiocchia, G. (2002a). Aeroelastic response of a 2-D airfoil in a compressible flow field and exposed to blast loading. Aerospace Science and Technology, 6, 259-272.

Marzocca, P., Librescu, L., and Chiocchia, G. (2002b). Aeroelasticity of two-dimensional lifting surfaces via indicial function approach. Aeronautical Journal, 106, 147-153.

Morse, A. S. (1993). A gain matrix decomposition and some of its applications. Systems and Control Letters, 21, 1-10.

Mukhopadhyay, V. (1992). Flutter suppression digital control law design and testing for the AFW Wind tunnel model. NASA Technical Memorandum, 107652.

Mukhopadhyay, V. (2000a). Benchmark active control technology: part 1. Journal of Guidance, Control, and Dynamics, 23, 913-960.

Mukhopadhyay, V. (2000b). Benchmark active control technology: part 2. Journal of Guidance, Control, and Dynamics, 23, 1093-1139.

Mukhopadhyay, V. (2001). Benchmark active control technology: part 3. Journal of Guidance, Control, and Dynamics, 24, 146-192.

Mukhopadhyay, V., Newsom, J. R., and Abel, I. (1980). Direct method for synthesizing low-order optimal feedback control laws with application to flutter suppression. Proceedings of the AIAA Atmospheric Flight Mechanics Conference, Danvers, MA. pp. 465-475 (AIAA-1980-1613).

Noor, A. K. and Venneri, S. L. (1992). Flight-Vehicle Materials, Structures, and Dynamics-Assessment and Future Directions. Vol. 5. Structural Dynamics and Aeroelasticity. New York: American Society of Mechanical Engineers. pp. 179-212.

Platanitis, G. and Strganac, T. W. (2004). Control of a Nonlinear Wing Section Using Leading- and Trailing-Edge Surfaces. Journal of Guidance, Control, and Dynamics, 27, 52-58.

Qin, Z., Marzocca, P., and Librescu, L. (2002). Aeroelastic instability and response of advanced aircraft wings at subsonic flight speeds. Aerospace Science and Technology, 6, 195-208.

Rao, V. M., Behal, A., Marzocca, P., and Rubillo, C. M. (2006). Adaptive aeroelastic vibration suppression of a supersonic airfoil with flap. Aerospace Science and Technology, 10, 309315.

Reddy, K. K., Chen, J., Behal, A., and Marzocca, P. (2007). Multi-input/multi-output adaptive output feedback control design for aeroelastic vibration suppression. Journal of Guidance, Control, and Dynamics, 30, 1040-1048.

Singh, S. N. and Wang, L. (2002). Output feedback form and adaptive stabilization of a nonlinear aeroelastic system. Journal of Guidance, Control, and Dynamics, 25, 725-732.

Strganac, T. W., Ko, J., Thompson, D. E., and Kurdila, A. J. (2000). Identification and control of limit cycle oscillations 
in aeroelastic systems. Journal of Guidance, Control, and Dynamics, 23, 1127-1133.

Strganac, T. W. and Mook, D. T. (1990). Numerical model of unsteady subsonic aeroelastic behavior. AIAA Journal, 28, 903-909.

Tao, G. (2003). Adaptive Control Design and Analysis. Hoboken: Wiley-Interscience.

Vasiljevic, L. K. and Khalil, H. K. (2008). Error bounds in differentiation of noisy signals by high-gain observers. Systems and Control Letters, 57, 856-862.

Vipperman, J. S., Clark, R. L., Conner, M., and Dowell, E. H. (1998). Investigation of the experimental active control of a typical section using a trailing-edge flap. Journal of Aircraft, 35, 224-229.

Wang, Z., Behal, A., and Marzocca, P. (2010). Modelfree control design for MIMO aeroelastic system subject to external disturbance. Journal of Guidance, Control, and Dynamics, accepted to be published.

Wazak, M. and Srinathkumar, S. (1992). Design and experimental validation of a flutter suppression controller for the active flexible wing. NASA Technical Paper, 4381.

Xing, W. H. and Singh, S. N. (2000). Adaptive output feedback control of a nonlinear aeroelastic structure. Journal of Guidance, Control, and Dynamics, 23, 1109-1116.

Yang, Z. C. and Zhao, L. C. (1988). Analysis of limit cycle flutter of an airfoil in incompressible flow. Journal of Sound and Vibration, 123, 1-13.

Zeng, Y. and Singh, S. N. (1998). Output feedback variable structure adaptive control of an aeroelastic system. Journal of Guidance, Control, and Dynamics, 21, 830-837.

Zhang, F. and Soffker, D. (2009). Active flutter suppression of a nonlinear aeroelastic system using PI-observer. In H. Ulbrich and L. Ginzinger, eds. Motion and Vibration Control: Selected Papers from MOVIC 2008. Dordrecht, The Netherlands: Springer.

Zhang, R. and Singh, S. N. (2001). Adaptive output feedback control of an aeroelastic system with unstructured uncertainties. Journal of Guidance, Control, and Dynamics, 24, 502-509. 